\title{
ARTICLE OPEN \\ Neonatal annulus fibrosus regeneration occurs via recruitment and proliferation of Scleraxis-lineage cells
}

\author{
Olivia M. Torre $\mathbb{D}^{1}$, Victoria Mroz ${ }^{1}$, Anthony R. Martinez Benitez ${ }^{1}$, Alice H. Huang (D) $^{1 *}$ and James C. latridis (D) ${ }^{1 *}$
}

Intervertebral disc (IVD) injuries are a cause of degenerative changes in adults which can lead to back pain, a leading cause of disability. We developed a model of neonatal IVD regeneration with full functional restoration and investigate the cellular dynamics underlying this unique healing response. We employed genetic lineage tracing in mice using Scleraxis (Scx) and Sonic hedgehog (Shh) to fate-map annulus fibrosus (AF) and nucleus pulposus (NP) cells, respectively. Results indicate functional AF regeneration after severe herniation injury occurs in neonates and not adults. AF regeneration is mediated by Scx-lineage cells that lose ScxGFP expression and adopt a stem/progenitor phenotype (Sca-1, days 3-14), proliferate, and then redifferentiate towards type I collagen producing, ScxGFP + annulocytes at day 56. Non Scx-lineage cells were also transiently observed during neonatal repair, including Shh-lineage cells, macrophages, and myofibroblasts; however, these populations were no longer detected by day 56 when annulocytes redifferentiate. Overall, repair did not occur in adults. These results identify an exciting cellular mechanism of neonatal AF regeneration that is predominantly driven by $S c x$-lineage annulocytes.

npj Regenerative Medicine (2019)4:23 ; https://doi.org/10.1038/s41536-019-0085-4

\section{INTRODUCTION}

Injured adult intervertebral discs (IVDs) heal poorly with the formation of a superficial, fibrous cap at the outermost layers of the annulus fibrosus (AF) and in some cases do not heal at all. ${ }^{1-3}$ Regeneration of the IVD remains an elusive goal due to its complex structure consisting of multiple, developmentally distinct tissues, challenging microenvironment, and continuous mechanical loading at high forces. IVDs are fibrocartilaginous joints that connect adjacent vertebrae and serve primarily mechanical roles to enable spinal motion and support high spinal forces. Important to the IVD's ability to serve these functions are its two major components, the AF and nucleus pulposus (NP). The AF is a collagen-rich, fibrocartilaginous structure that forms the outer ring of the IVD; the tough, ligament-like AF fibers constrain the centrally-located NP, which is a proteoglycan-rich cartilaginous structure with high swelling propensity. Acute injury or degenerative changes to the AF are commonly associated with IVD herniation, which is defined as extrusion of NP tissue through an AF defect. Herniations can involve radiculopathy (lower back and leg pain and disability) caused by compression and irritation of the NP on adjacent nerve roots. When conservative measures fail to reduce pain and disability from radiculopathy, surgical discectomy is used. In the United States, 300,000-400,000 patients receive discectomy procedures ${ }^{4}$ which treat the acute nerve root compression injury by surgically removing herniation tissue without repairing AF defects. Discectomy has more favorable outcomes than non-operative treatment in most patients, but unrepaired AF defects can contribute to recurrent or persistent radiculopathy, reherniation in up to $27 \%$ of patients, and accelerated IVD degeneration. ${ }^{5-7}$ Despite the prevalence of postdiscectomy complications, treatment options are limited with varied outcomes and no effective AF repair strategies. Therefore, there is an unmet clinical need for the development of regenerative AF repair strategies that restore IVD structure and mechanical function.
Although adult mammals can efficiently regenerate some tissues such as liver, ${ }^{8,9}$ bone, $^{10,11}$ and muscle, ${ }^{12,13}$ for most tissues (such as heart, pancreas, and nerves ${ }^{14}$ ), regenerative capacity is absent and the default mode of healing is fibrotic scar formation. Regeneration of normally non-regenerative tissues is therefore typically studied using model organisms (such as axolotls and zebrafish) or more rarely, in specific mouse strains (such as MRL and African spiny mice). Recently, the neonatal mouse has also emerged as an exciting new model of mammalian regeneration that is not limited to a particular mouse strain. To date, the capacity for neonatal regeneration has been demonstrated for diverse tissues including the heart, $^{15}$ cochlea, $^{16}$ digit tip, ${ }^{17}$ tendon, ${ }^{18}$ and IVD. ${ }^{19}$

The adult IVD is normally non-regenerative since AF injury leads to several pathological changes, including decreased IVD cellularity, matrix degeneration, innervation, inflammation, and formation of granulation tissue. ${ }^{20,21}$ Poor healing is generally thought to be a consequence of limited vascularity and the mechanically challenging microenvironment. However, we recently showed that neonatal IVDs are capable of functional regeneration following severe herniation injury, with restored IVD height, biomechanical properties, and improved structural healing compared to adults. ${ }^{19}$ Furthermore, using the transgenic ScxGFP reporter, which identifies tenocytes and AF cells (annulocytes), ${ }^{22}$ we observed a population of SCXGFP-negative cells occupying the injury site one month after herniation, ${ }^{19}$ suggesting either annulocyte dedifferentiation or recruitment of non Scx-lineage (non AF-derived) cells. To address this question, we now identify the cellular players and their dynamics during neonatal and adult AF healing using inducible genetic lineage tracing of Scx-lineage (AF-derived, Scxlin) and Shh-lineage (NP-derived, Shh-lin) cells. We found that neonatal AF regeneration is primarily driven by $S C x$-lin cells that lose ScxGFP expression and adopt a stem/progenitor phenotype. Following recruitment, $S c x$-lin cells expand via proliferation and reacquire ScxGFP expression and markers consistent with an

\footnotetext{
${ }^{1}$ Leni \& Peter W. May Department of Orthopaedics, Icahn School of Medicine at Mount Sinai, One Gustave Levy PlaceBox 1188, New York, NY 10029-6574, USA.

*email: alice.huang@mssm.edu; james.iatridis@mssm.edu
} 
annulocyte fate. Non Scx-lin cells detected during AF regeneration included Shh-lin cells, macrophages, and myofibroblasts. However, the presence of these cells is transient. Collectively, these findings identify a key cellular mechanism underlying neonatal AF regeneration and may inform regenerative strategies for improving adult AF healing.

\section{RESULTS}

Early proliferation and minimal apoptosis following neonatal herniation

To establish cellular dynamics in the normal neonatal AF and following neonatal injury, proliferation and apoptosis was determined using EdU and TUNEL detection, respectively. In uninjured animals, annulocytes showed high proliferative capacity during early postnatal stages, with an early proliferative peak observed during the first week of age. ${ }^{23}$ Neonatal IVDs were therefore injured within the first week of age at p5 using the ScxGFP reporter to visualize IVD structures directly through the skin. Injury was created using a dorsal-lateral approach with a beveled syringe needle tip inserted to $50 \%$ of IVD diameter (Fig. $1 \mathrm{a}, \mathrm{b})$. A near-immediate increase in proliferative activity was observed 2 hours (hrs) after injury, with $\sim 14 \%$ of cells proliferating in the injured $\mathrm{AF}$, compared to $\sim 8 \%$ cell proliferation in uninjured, internal AF controls. Proliferation was maintained at day 3 postinjury (d3), with $\sim 13 \%$ of cells proliferating in injury relative to $\sim 7 \%$ in controls (Fig. 1c, d). Proliferating cells were also observed in regions near to the injury site, including the growth plates and connective tissue adjacent to the outer $\mathrm{AF}$, at both day 0 and day 3. TUNEL staining showed minimal apoptosis in all samples, regardless of injury or timepoint (Fig. 1e, f). These results suggest that a rapid proliferative response in neonates may be an early driver of regenerative healing.

\section{Neonatal injury sites are populated by Scx-lin and non Scx-lin} ScxGFP+ annulocytes

To determine the dynamics of AF-specific differentiation after injury, ScxGFP expression was determined at early (d3), middle (d28), and late (d56) timepoints after neonatal and adult injury. In neonates at $\mathrm{d} 3$, the injured AF site was highly cellularized with little ScxGFP expression in these cells relative to uninjured controls (Fig. 2a). At d28, the injured AF remained highly populated by SCXGFP-cells that were immediately adjacent to ScXGFP + cells of the intact $A F$, identified by its organized lamellar structure (Fig. $2 b)$. At d56, which is when functional biomechanical properties are restored in neonates, ${ }^{19}$ ScxGFP+ cells were detected within the injury site, indicative of annulocyte differentiation; however cell density in control and injured IVDs was reduced compared to previous timepoints. Although ScxGFP expression in cells of the adjacent, intact $A F$ region was relatively low, this is consistent with low SCXGFP expression in the non-injured control AF, in which ScXGFP can only be detected in outer annulocytes (Fig. 2c). This downregulation/restriction of ScxGFP expression may coincide with the end of AF tissue growth (similar to previous reports in tendon ${ }^{18}$ ) or downregulation of SCXGFP in inner annulocytes, which are more chondrogenic. ${ }^{24}$

In contrast to neonatal recellularization and differentiation of ScxGFP+ annulocytes, the injury site of adult AF was largely devoid of extracellular matrix and cells (Fig. $2 \mathrm{~d}-\mathrm{g}$ ). Alcian Blue/Fast red staining for cartilage and cell nuclei, respectively, revealed that the neonatal injury site was occupied by repair extracellular matrix and was highly cellular, in contrast to the adult injury site which was occupied by minimal repair tissue and was minimally cellularized, and where healing was limited to a cellular cap at the periphery of the injury site (Fig. 2g). Quantification of the few cells present showed that the percentage of ScxGFP+ cells in injured adult IVDs at d56 (7\% ScxGFP+) was significantly less than in d56 neonates (53\% ScxGFP+) (Fig. 2h).

Annulocyte differentiation in neonates was further assessed by AF-specific gene expression of Scx, Tnmd, Mkx, and Col1a1 at d3 and d56 in uninjured, control IVDs and injured IVDs. In neonates, Tnmd, Mkx, and Collal gene expression were unchanged in injured IVDs compared to controls at d3 or d56, while $S c x$ expression was increased at d56 (Supplemental Fig. 1). Tenogenic genes were also unaffected in adults. As expected, Col2a1 expression was relatively low in all groups, indicating successful removal of NP tissue during dissection and absence of aberrant cartilage differentiation with healing. Scar-associated marker Fn1 was not affected after injury in both neonates and adults. While it is surprising that AF-specific markers are mostly unchanged overall, it is possible that whole IVD analysis is not sufficiently sensitive to detect changes occurring in the injury site.

Annulocytes in neonatal regeneration are derived from Scx-lin and non Scx-lin sources

To identify the source of IVD repair cells, we next determined whether differentiation of ScxGFP+ annulocytes was mediated by recruitment of $S c x$-lin cells with mitotic potential or non Scx-lin cells (potentially stem cells or other wound healing cells). Lineage tracing of Scx-lin annulocytes was carried out using ScxCreERT2 and cells labeled by tamoxifen prior to injury (Fig. 3a). Using TdTomato and SCXGFP expression, four distinct populations were identified: $S c x$-lin/ScxGFP- cells (Scx-lin cells), ScxGFP+ cells (extrinsically recruited annulocytes), Scx-lin/ScxGFP+ cells (Scx-lin annulocytes), and DAPI only cells (Fig. 3b). Within the neonatal injury site, most recruited cells were $S c x$-lin cells derived from the original AF ( 54\% Scx-lin+), and the majority of these Scx-lin cells also expressed ScxGFP+. A smaller population of ScxGFP+ only cells was also observed, derived from non Scx-lin sources $(\sim 18 \%$, Fig. 3d). By contrast, few cells within the adult injury site were Scxlin $(\sim 3 \%)$ or ScxGFP+ ( 7\%), and significant differences were observed for almost all cell populations relative to neonates (Fig. 3e). Scx-lin annulocytes were observed in the neonatal injury site $(\sim 40 \%$, Fig. $3 f)$ in contrast to adults where no Scx-lin annulocytes were observed. Interestingly, a smaller population of extrinsically recruited annulocytes were observed in neonates ( 18\%, Fig. $3 g$ ), and the few ScxGFP+ cells observed in the adult injury site were non $S c x$-lin, although these cells could also be accounted for by incomplete recombination efficiency. Of the few cells observed in the adult injury site, a majority were DAPI only cells ( $90 \%$, Fig. $3 \mathrm{~h})$ compared to neonates that had a smaller population of DAPI only cells. Together, these findings suggest that recruitment and differentiation of Scx-lin annulocytes is a distinctive feature of neonatal AF healing and may drive functional regeneration after injury.

Loss of ScxGFP and proliferation of Scx-lineage annulocytes precedes recruitment and redifferentiation

To determine the temporal dynamics of Scx-lin cell recruitment, annulocytes were labeled at p1-p3 and traced at 2 hrs, d3, d14, and d56 (Fig. 4a). In uninjured control IVDs, all annulocytes were $S c x G F P+$. Labeling of Scx-lin annulocytes was incomplete at $2 \mathrm{hrs}$ (Fig. 4b), with improved recombination efficiency at subsequent timepoints (Fig. 4c-e). At $2 \mathrm{hrs}$, infiltration of DAPI only cells were observed in the puncture tract (Fig. 4b). At d3, the expanded injury site was occupied by a few Scx-lin cells, but none of these cells were ScxGFP+, suggesting that Scx-lin annulocytes had dedifferentiated (Fig. 4c). Increasing numbers of Scx-lin cells were observed at d14, although these cells remained ScxGFP- (Fig. 4d). By d56, most cells in the injury site were differentiated ScXGFP+ annulocytes derived from Scx-lin and non Scx-lin cells (Fig. 4e).

To determine whether Scx-lin cells observed at the d3 and d14 injury site were potentially dedifferentiated annulocytes adopting 
A
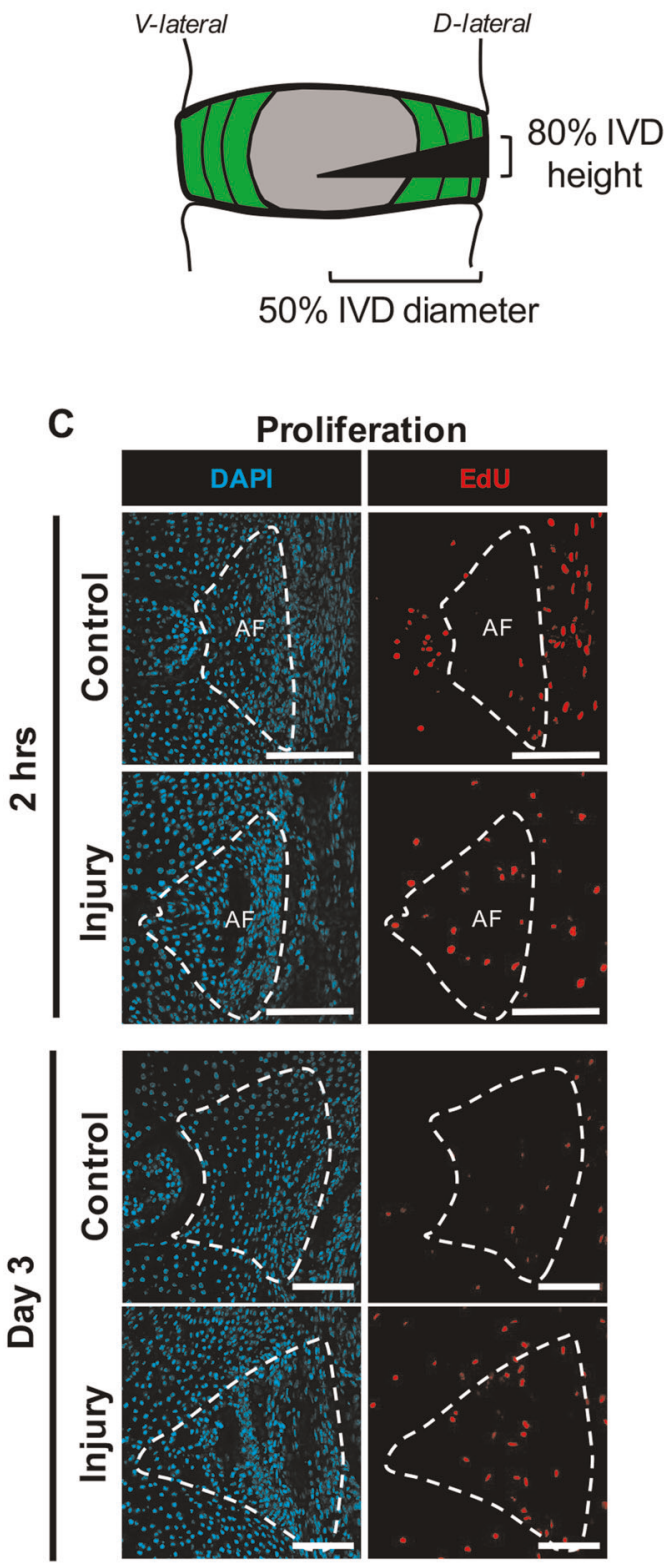

D

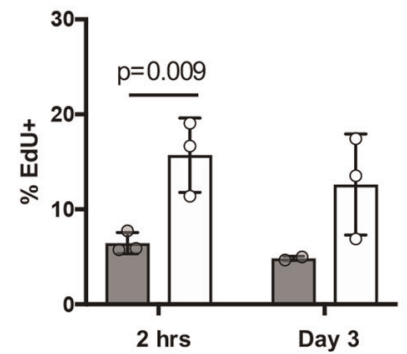

B

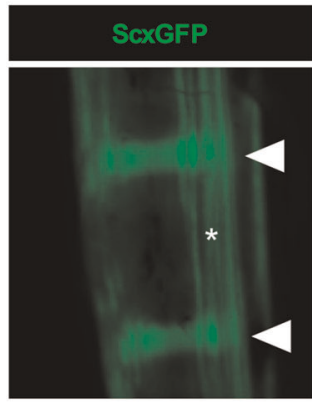

E
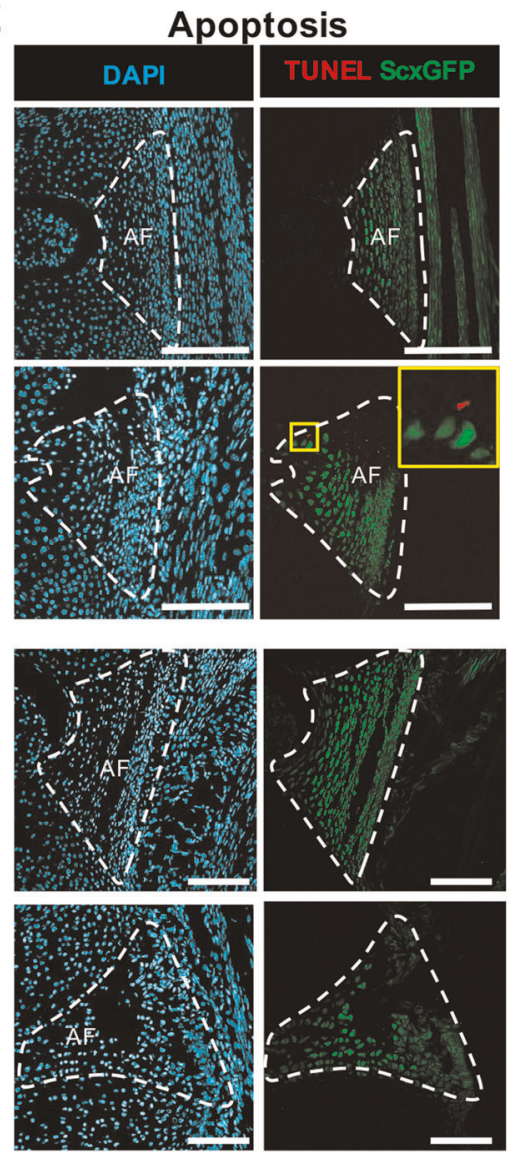

$\mathbf{F}$

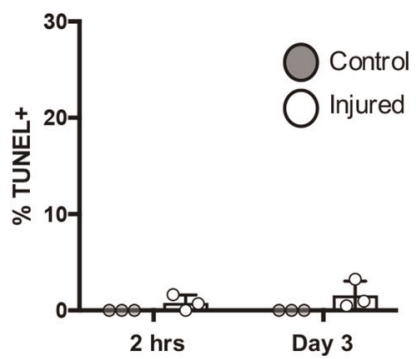

Fig. 1 Early proliferation and minimal apoptosis of injury site cells occurs immediately following neonatal herniation. Neonatal injuries were performed using a 31G beveled syringe needle tip to 50\% of the IVD diameter a in the ScxGFP reporter mouse that labels annulocytes (triangles) and tenocytes (asterisks) b. ScxGFP expression is decreased in the neonatal IVD 2 hrs post-injury (yellow triangle) b. Proliferating cells were detected using EdU. Representative images of the posterior control AF of an uninjured IVD and the posterior AF of an injured IVD show an increase in proliferation at $2 \mathrm{hrs}$ and $\mathrm{d} 3 \mathrm{c}$. Quantification using cell counting determined that there was a significant increase in the percentage of proliferating cells at d0 ( 2 hrs post-injury) compared to controls $\mathbf{d}$. Cells undergoing apoptosis were detected using TUNEL staining. Minimal apoptosis was observed in uninjured controls at $2 \mathrm{hrs}$, where the few cells stained positive for TUNEL were located at the border of ScxGFP + cells and ScxGFP-cells in the injured AF and at d3 e. No differences between the percentage of TUNEL-positive cells were observed between control and injured AFs f. Error bars $=$ SD. Scale $=100 \mu \mathrm{m}$. 


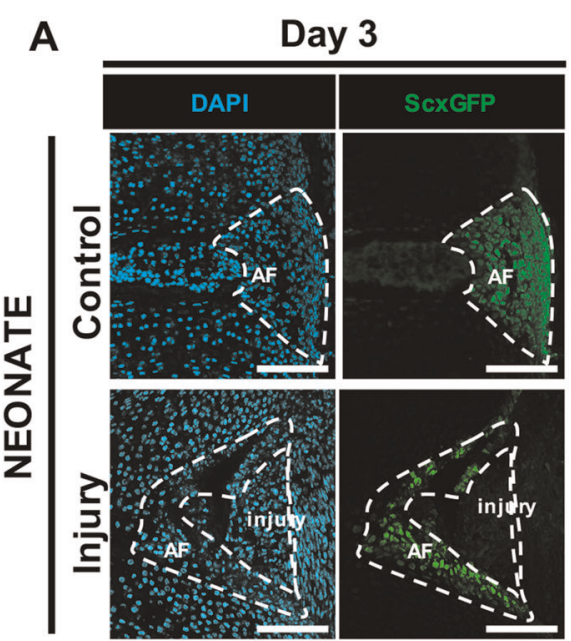

B

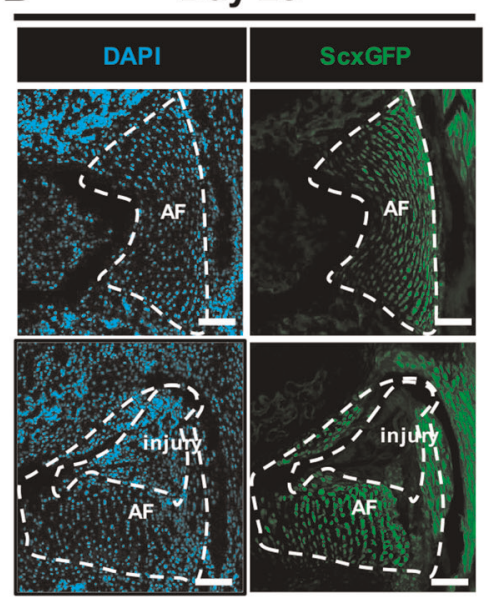

E

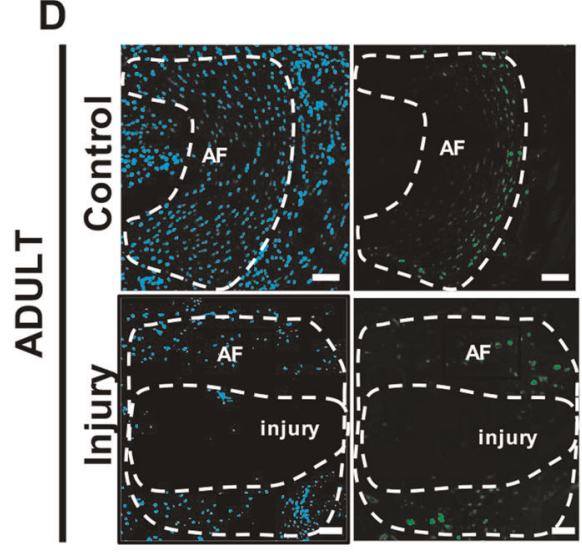

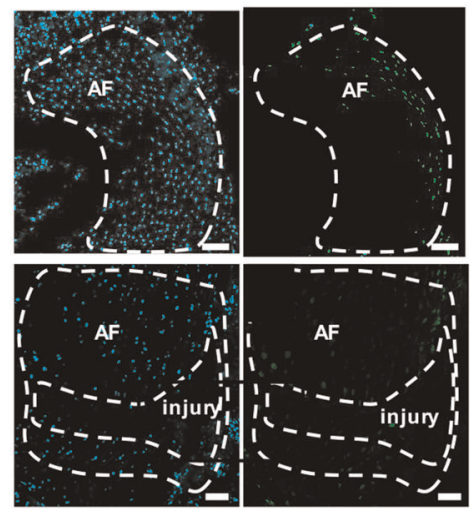

C

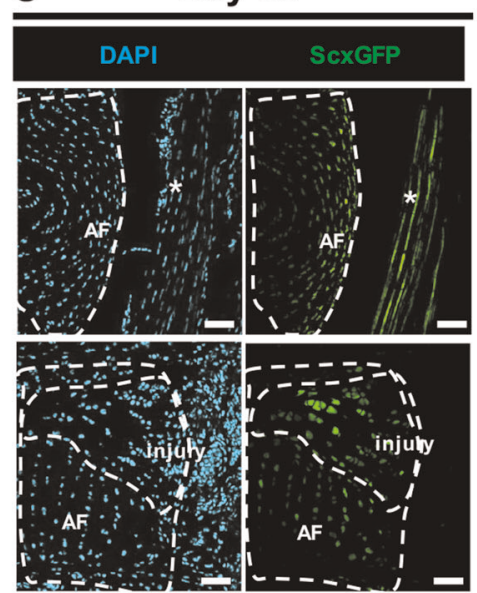

F

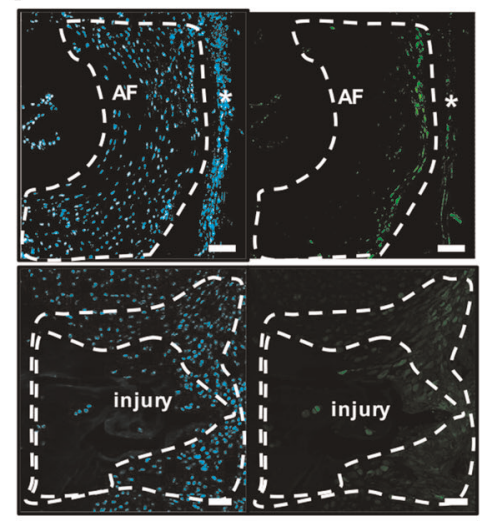

$\%$ cells / total \# DAPI

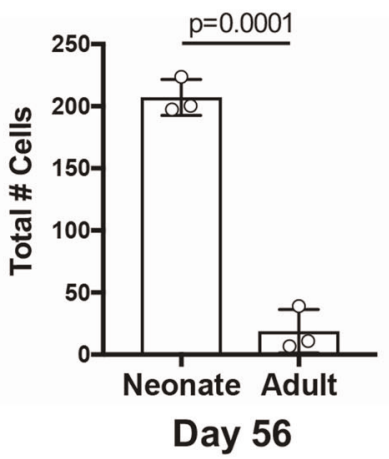

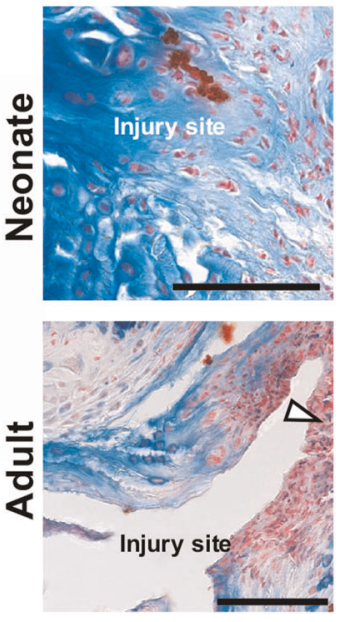
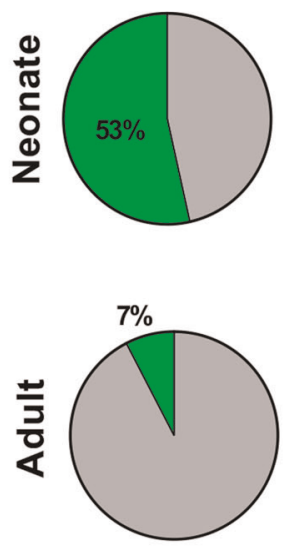

ScxGFP+
H

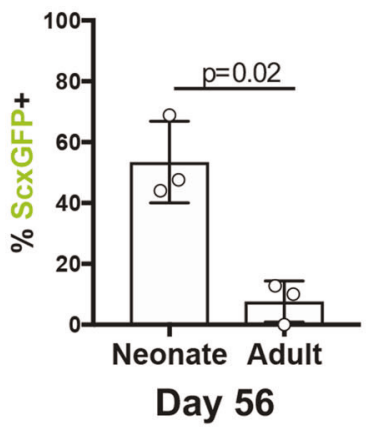

ScxGFP-

Fig. 2 ScxGFP annulocyte differentiation occurs in the neonatal injury site by d56. ScxGFP expression is observed in the uninjured, control $A F$ in almost all cells at d3 a. ScxGFP expression is decreased in the inner AF at d28 b, and is limited to the outer AF by d56 c and at all adult stages d-f. At d3 following injury, the neonatal injury site is cellular but most cells in the injury site are ScxGFP-a. At d28, the injury site remains cellular and consists of SCXGFP- cells immediately adjacent to ScxGFP + cells that appear to be from intact AF and are organized into aligned layers b. At d56, ScxGFP expression in annulocytes adjacent to the injury site appears decreased, and cells within the injury site express ScxGFP c. The adult injury site has minimal cellularity at $\mathrm{d} 3, \mathbf{d} 28$, and $\mathrm{d} 56(\mathbf{d}-\mathbf{f})$. The neonatal injury site was occupied by extracellular matrix and was highly cellularized, while in adults, the injury site remained largely void of extracellular matrix or cells, and a fibrous, cellular cap lined the periphery of the posterior AF (G, white triangle). The total number of cells recruited to the injury site was increased in neonates compared to adults g. ScxGFP expression at the d56 is restored in $\sim 53 \%$ of cells of the injury site in neonates, compared to $7 \%$ in adults $\mathbf{h}$. Adjacent tendon $\left.{ }^{*}\right)$. Error bars $=$ SD. Scale $=100 \mu \mathrm{m}$. 
A ScxCreERT2/ScxGFP/RosaT

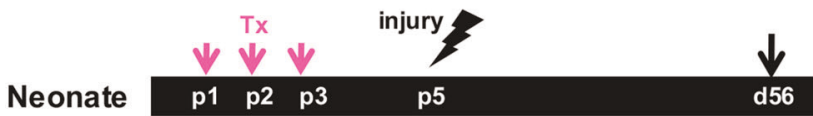

B 3 sagittal sections / IVD

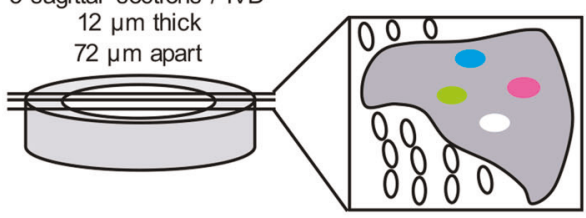

ScxCET+/ ScxGFP- : Scx-lin cell ScxCET+I ScxGFP+ : Scx-lin annulocyte ScxCET- / ScXGFP+: non Scx-lin annulocyte ScxCET-/ ScxGFP- : DAPI only cell
C

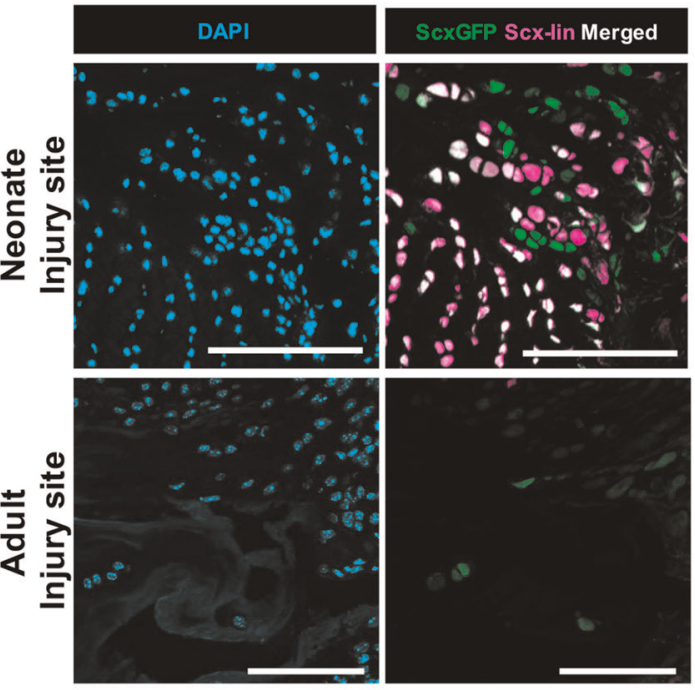

E

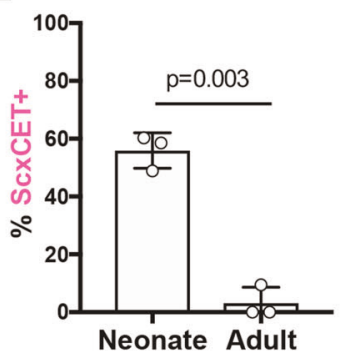

$\mathbf{F}$

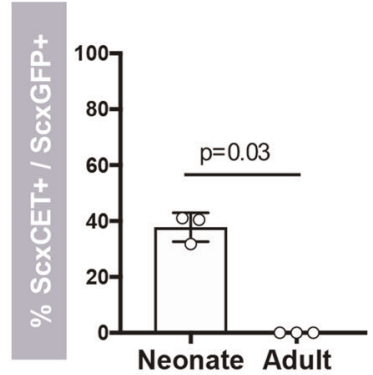

G

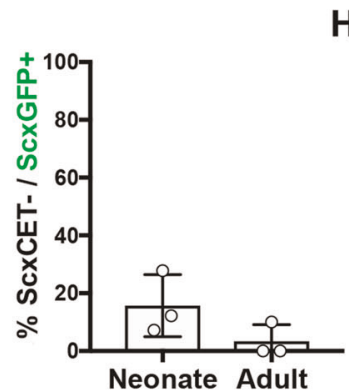

$\%$ cells / total \# DAPI

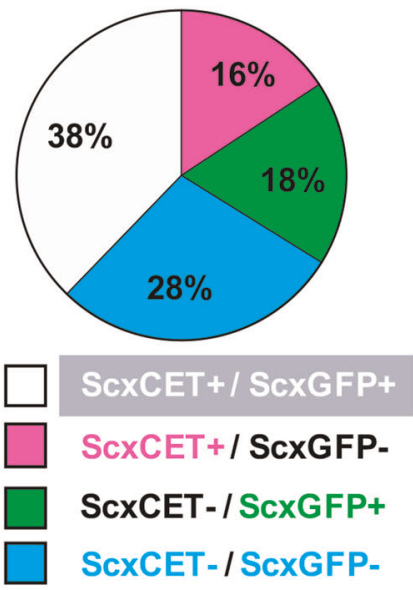

H

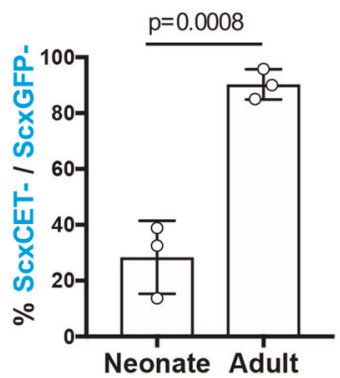

Fig. 3 Restoration of ScxGFP expression in Scx-lin and non Scx-lin annulocytes occurs following injury in neonates. ScxCreERT2/RosaT/ ScXGFP mice were used to trace the fate of annulocytes after herniation in neonates and adults. Annulocytes were labeled by tamoxifen delivery at $\mathrm{p} 1-\mathrm{p} 3$ in neonates or 1 week prior to injury in adults, followed by injury at $\mathrm{p} 5$ in neonates and p112 in adults, and subsequent lineage tracing of $S c x$-lin annulocytes at d56 a. Four distinct populations of cells were observed in the AF injury site and quantified by averaging three consecutive sections, and include Scx-lin cells that are not annulocytes (ScxCET+/ScxGFP-), Scx-lin annulocytes (ScxCET $+/ S c x G F P+$ ), non Scx-lin annulocytes (ScxCET-/ScxGFP+), and non Scx-lin cells labeled with DAPI only (ScxCET-/ScxGFP-) b. The neonatal injury site was occupied by differentiated ScxGFP annulocytes that were also Scx-lin cells while the adult injury site was largely devoid of cells c. In neonates, the largest population of cells in the injury site were $S c x$-lin, of which $38 \%$ were $S c x$-lin $(S c x C E T+)$ annulocytes and $16 \%$ were $S c x$-lin and no longer expressed ScxGFP. The next largest population of cells were $18 \%$ of cells were neither Scx-lineage nor ScxGFP+ (DAPI only), followed by the smallest population of cells that were non Scx-lin (ScxCET-) annulocytes d. The percentages of Scx-lin annulocytes and non Scxlin annulocytes were significantly decreased in adults compared to neonates at d56 e-g. The percentage of SCXCET-/ScxGFP- cells (DAPI only) in the d56 injury site was significantly greater in adults compared to neonates $\mathbf{h}$. Error bars $=\mathrm{SD}$. Scale $=100 \mu \mathrm{m}$.

a stem/progenitor phenotype, co-localization of stem cell antigen 1 (Sca-1) with Scx-lin cells was assessed (Fig. 5). At d3, Sca-1 expressing cells were broadly observed at the injury site. These cells were mostly ScxGFP- and the few Scx-lin cells present colocalized with Sca-1 (Fig. 5a). At d14, ScxGFP- cells occupied the injury site and Scx-lin cells co-localized with Sca-1 (Fig. 5b). Cells that expressed Sca-1 but did not co-localize with either ScxGFP or
Scx-lin cells were also observed, potentially indicating recruitment of a stem/progenitor like cell to the injury site. At d56, Sca-1 was present in the injury site but appeared decreased compared to d3 and $\mathrm{d} 14$ while ScxGFP+ cells were increased (Fig. 5c). Subpopulations of Sca-1 expressing cells were observed at d56 including Scxlin/ScxGFP+ cells, non Scx-lin/ScxGFP+ cells, and non Scx-lin/ SCXGFP- cells. These findings suggest that ScX-lin annulocytes 


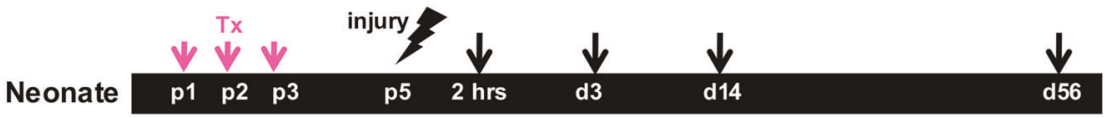

B
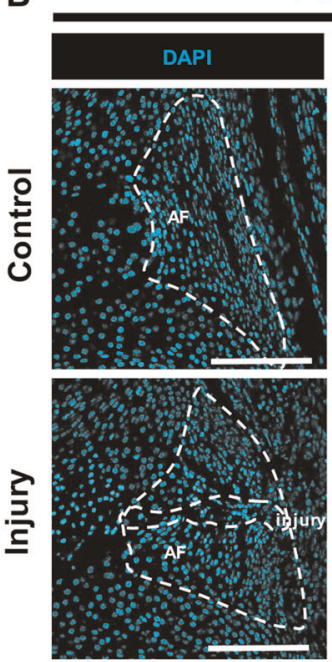

D
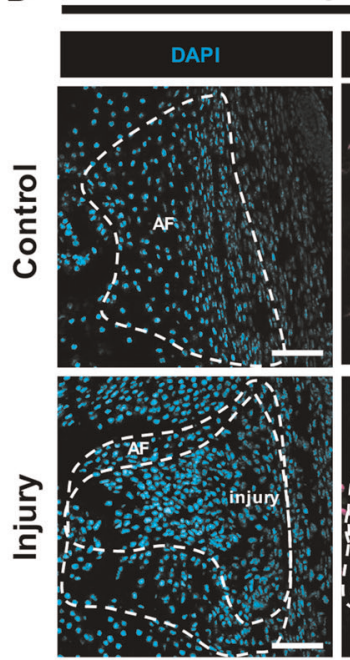

$2 \mathrm{hrs}$
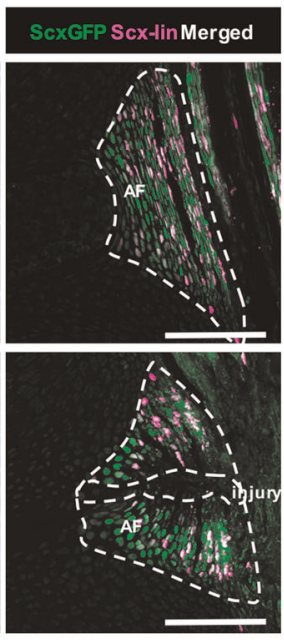

Day 14
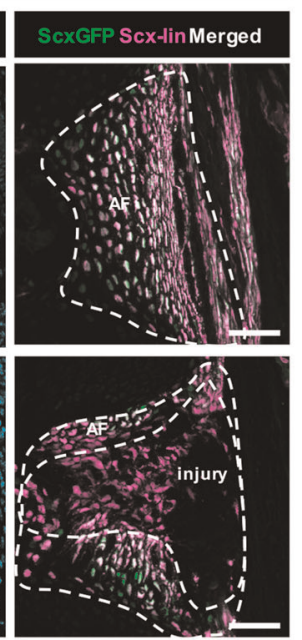

C

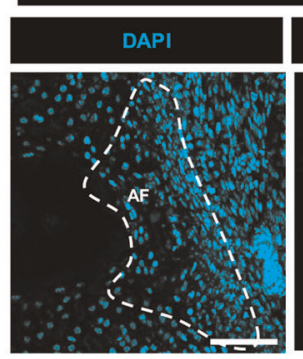

Day 3

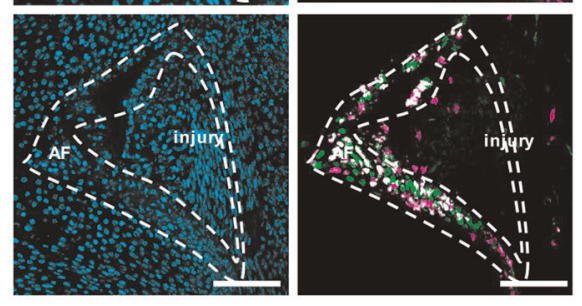

E
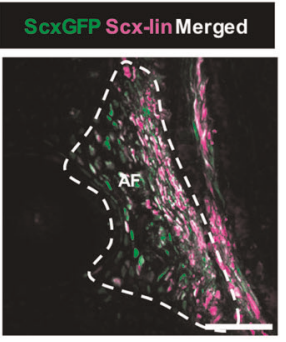

Day 56
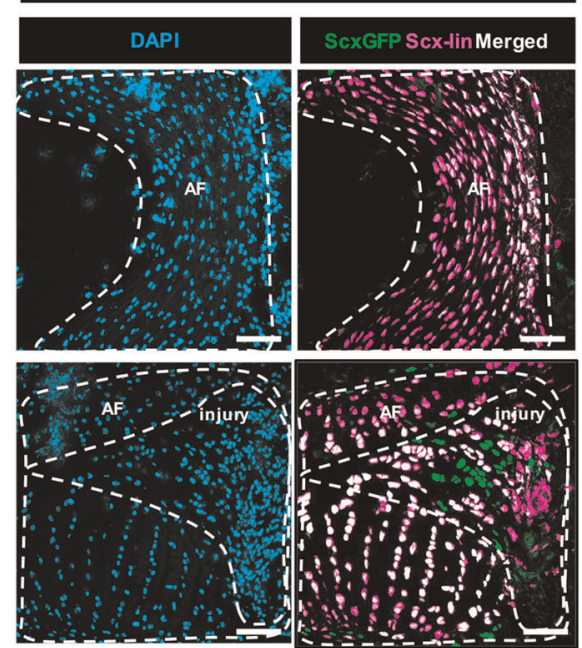

Fig. 4 Neonatal AF healing occurs via a transient loss of ScxGFP in Scx-lin annulocytes followed by restoration of ScxGFP. ScxCreERT2/ RosaT/ScXGFP mice were used to trace the fate of annulocytes that were labeled with tamoxifen at p1-p3, and traced after injury at 2 hrs, d3, d14, and d56 a. Scx-lin cells in the uninjured control AF were more numerous over time, with a smaller portion of annulocytes labeled at 2 hrs b compared to d56 where most annulocytes were Scx-lin e. At 2 hrs, the injury site was cellular but cells immediately adjacent to the puncture tract lost ScxGFP expression and were not Scx-lin b. At d3, the injury site expanded and contained cells that were neither ScxGFP+ nor Scx-lin c. At d14, the injury site remained ScxGFP-, and was occupied by Scx-lin cells recruited to the injury site d. By d56, differentiation of Scx-lin cells was observed by co-localization with ScxGFP e. Images were acquired at 20X and digitally magnified to show the entire posterior AF at each timepoint. Since IVDs are growing at these early stages, the length of the scale bar is decreasing with increasing timepoint. Scale $=100 \mu \mathrm{m}$.

acquire a stem/progenitor like phenotype following injury and points towards contributions from non Scx-lin stem/progenitor cells that are recruited to the injury site as early as $\mathrm{d} 3$.

Annulocytes are known to produce type-I collagen (Col I) with a decreasing gradient of expression from the outer AF towards the NP. Col I is therefore a useful marker and the gold standard for determining annulocyte phenotype. Immunostaining for Col I revealed that $S c x$-lin cells in the injury site at d3 were ScxGFP- and did not express Col I (Fig. 6a). At d14, Scx-lin cells colocalized with Col I (Fig. 6b). By d56, ScxGFP expression was restored and was colocalized with Col I (Fig. 6c). Together, the data suggest that ScxGFP expression in annulocytes is lost immediately following neonatal injury but annulocyte phenotype is likely restored by d56 as evidenced by colocalization of ScxGFP expression and Col I.

To determine whether expansion of Scx-lineage cells observed in the AF injury site was driven by proliferation of dedifferentiated, Scx-lin annulocytes or continuous recruitment, EdU was injected $2 \mathrm{hrs}$ prior to harvest (Fig. 7a). In control IVDs, Scx-lin cells comprised most proliferating cells at all timepoints ( 60-70\%, Fig. 7). Although Scx-lin cell proliferation decreased $2 \mathrm{hrs}$ after 
A
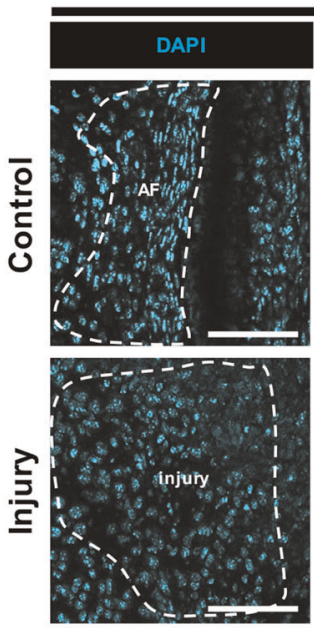

B

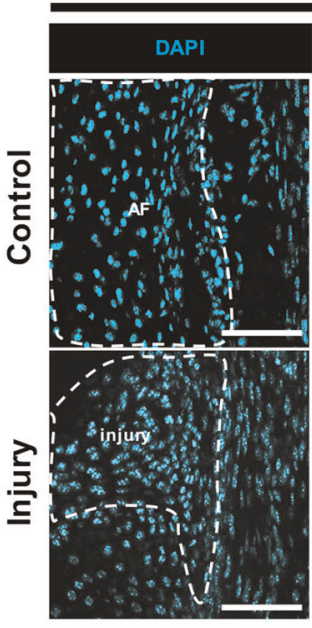

C
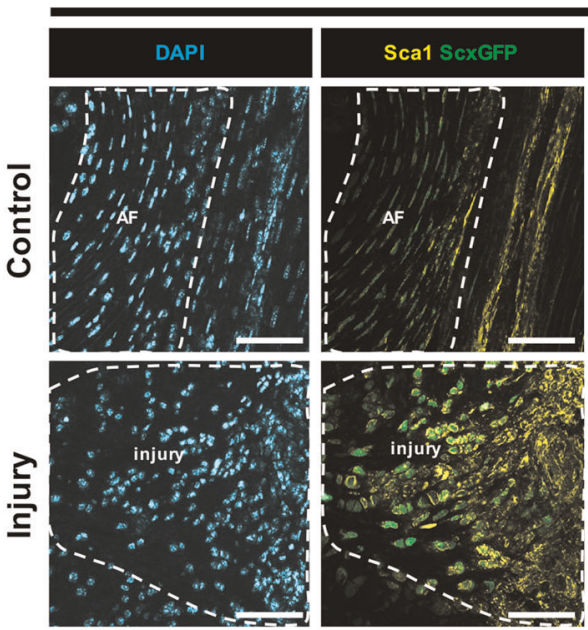
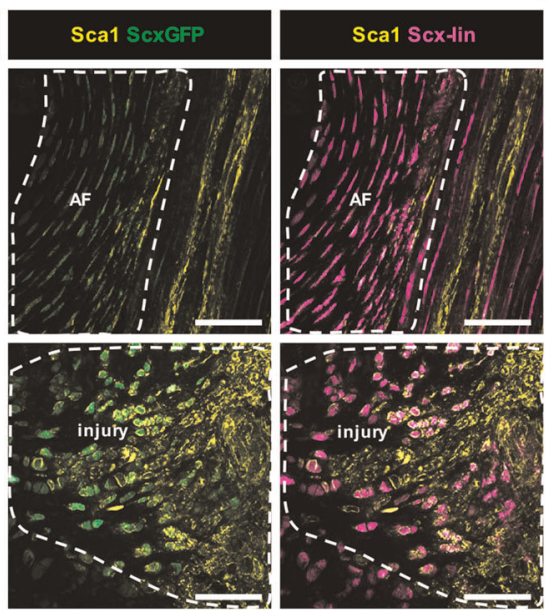

Sca1 Scx-lin
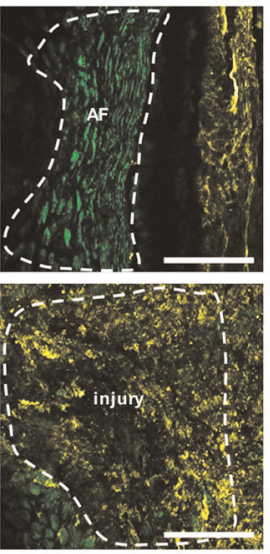

Day 14
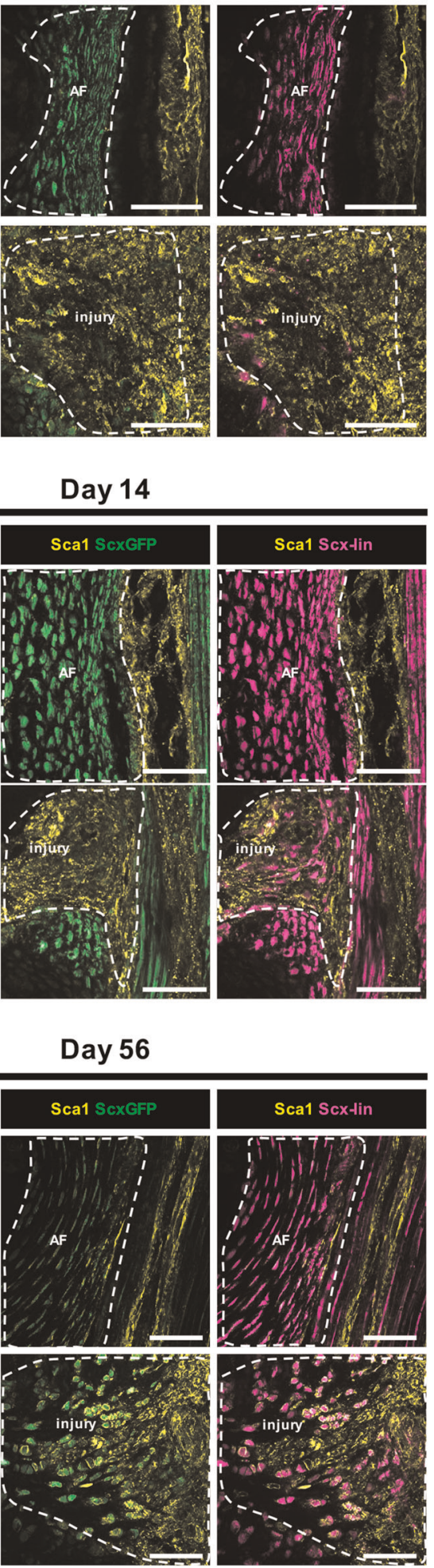

injury relative to controls (Fig. 7b, e), proliferation was improved at d3 and restored by d14 (Fig. $7 \mathrm{c}-\mathrm{g}$ ). At 2 hrs, most proliferating cells reside outside of the injury site, while at $d 3$ and $d 14$, proliferating cells are largely restricted to the injury site. Together, these findings suggest that Scx-lin annulocytes dedifferentiate

Fig. 5 Neonatal Scx-lin annulocytes lose ScxGFP expression and adopt a stem/progenitor phenotype following injury. Sca-1 immunostaining for stem/progenitor cells at $\mathrm{d} 3$ revealed broad presence of non Scx-lin cells throughout the injury site and colocalization with few Scx-lin cells a. Scx-lin cells colocalized with Sca-1 at d 14 b and d56 c with decreased Sca- 1 expression at the d56 injury site compared to $\mathrm{d} 3$ and d14. The presence of non Scx-lin, ScxGFP +, Sca- 1 expressing cells at day 56 points towards contributions of non $S c x$-lin stem/progenitors that are potentially recruited to the injury site and differentiate to a ScxGFP cell phenotype. Scale $=100 \mu \mathrm{m}$.

after injury with rapid recruitment into the injury site by $\mathrm{d} 3$. Expansion of this population is likely driven by proliferation with redifferentiation by $\mathrm{d} 56$.

Shh-lineage cells are transiently recruited to the AF injury site Although $S c x$-lin cells comprised most cells in the injury site at d56, non Scx-lin cells were also observed. One potential source of non Scx-lin cells may be herniated NP cells. Therefore, to determine whether NP cells are retained in the injury site or undergo transdifferentiation to annulocytes, lineage tracing was carried out using ShhCre (Fig. 8a). Although ShhCre is a wellestablished Cre driver for the NP, we were surprised to observe sporadic Shh-lin cells in the AF of uninjured IVDs (Fig. 8b-e). After injury, Shh-lin cells were no longer detected in the NP region, consistent with our previous findings indicating complete NP loss following puncture. At d3, a few Shh-lin cells with flattened, stellate morphology were observed in the injured AF (Fig. 8b); Shh-lin cells were still detected at d56 (Fig. 8c), but the low numbers of these cells suggest minimal contribution of Shh-lin cells to regeneration. Immunostaining for the mature NP cell marker cytokeratin 19 (CK19) did not show mature NP cells in the AF injury site (Supplemental Fig. 2). The presence of Shh-lin cells may therefore be due to NP cell dedifferentiation, retention of the sporadically labeled Shh-lin annulocytes, or Shh-lin cell recruitment from a non-NP source. In the adult injury site, Shh-lin cells were not detected at any timepoint (Fig. 8d, e).

The stellate morphology of Shh-lin cells in the neonatal d3 injury site was not consistent with the typical rounded morphology of NP cells. Since some reports indicate Shh signaling may activate myofibroblasts, ${ }^{25,26}$ we immunostained for myofibroblast marker a-SMA in ShhCre IVDs. Immunostaining showed no overlap, suggesting that these stellate Shh-lin cells are not myofibroblasts (Supplemental Fig. 3). We also screened for other cell types potentially involved in healing, using established markers for macrophages (F4/80), stem/progenitor cells (nucleostemin), pericytes (MCAM/CD146), myofibroblasts (a-SMA), and endothelial cells (PECAM/CD31). Although macrophages and myofibroblasts were transiently recruited to the injury site at early stages, pluripotent stem cells, endothelial cells, and pericytes were not detected (Supplemental Fig. 2). These findings suggest that Shh-lin cells, macrophages, and myofibroblasts comprise part of the non Scx-lin population during the early phase of neonatal regeneration, however other cells remain unidentified. These results demonstrate a potential role for a population of Shh-lin wound healing cells in the early AF injury response.

\section{DISCUSSION}

This study showed that neonatal AF regeneration is mediated by proliferation, recruitment, and restoration of SCXGFP and Col-I in Scx-lin annulocytes as well as non Scx-lin cells that adopt the annulocyte fate. The timecourse for AF differentiation was much longer (56 days) than regeneration of other neonatal tissues (14 or 21 days). ${ }^{18,27}$ This unexpectedly long differentiation process may 


\section{A}

Day 3
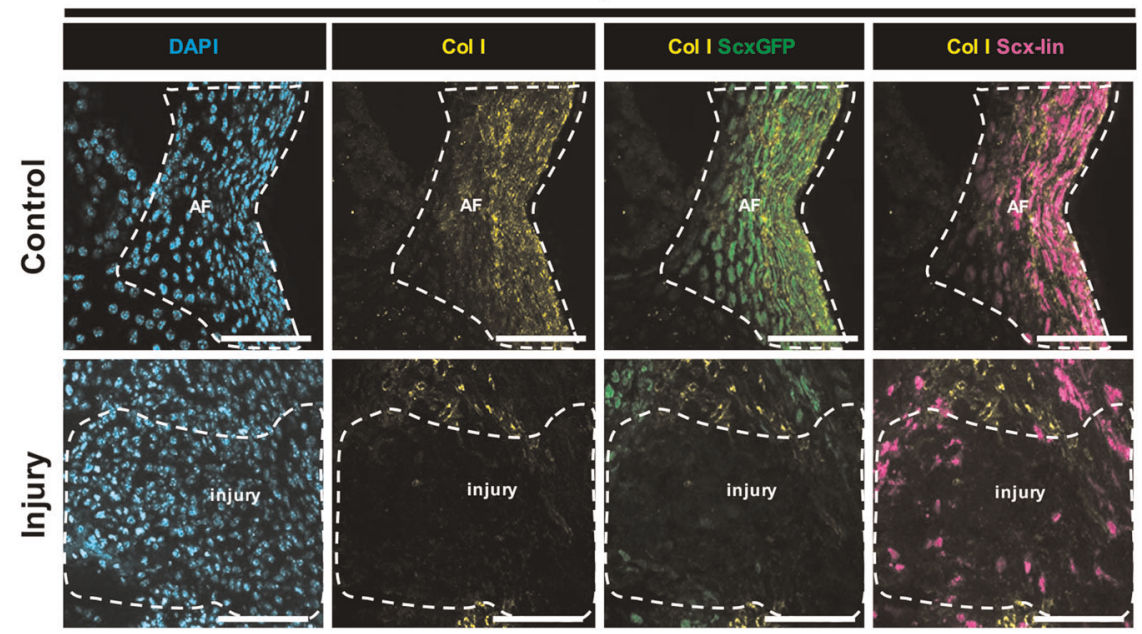

B

Day 14
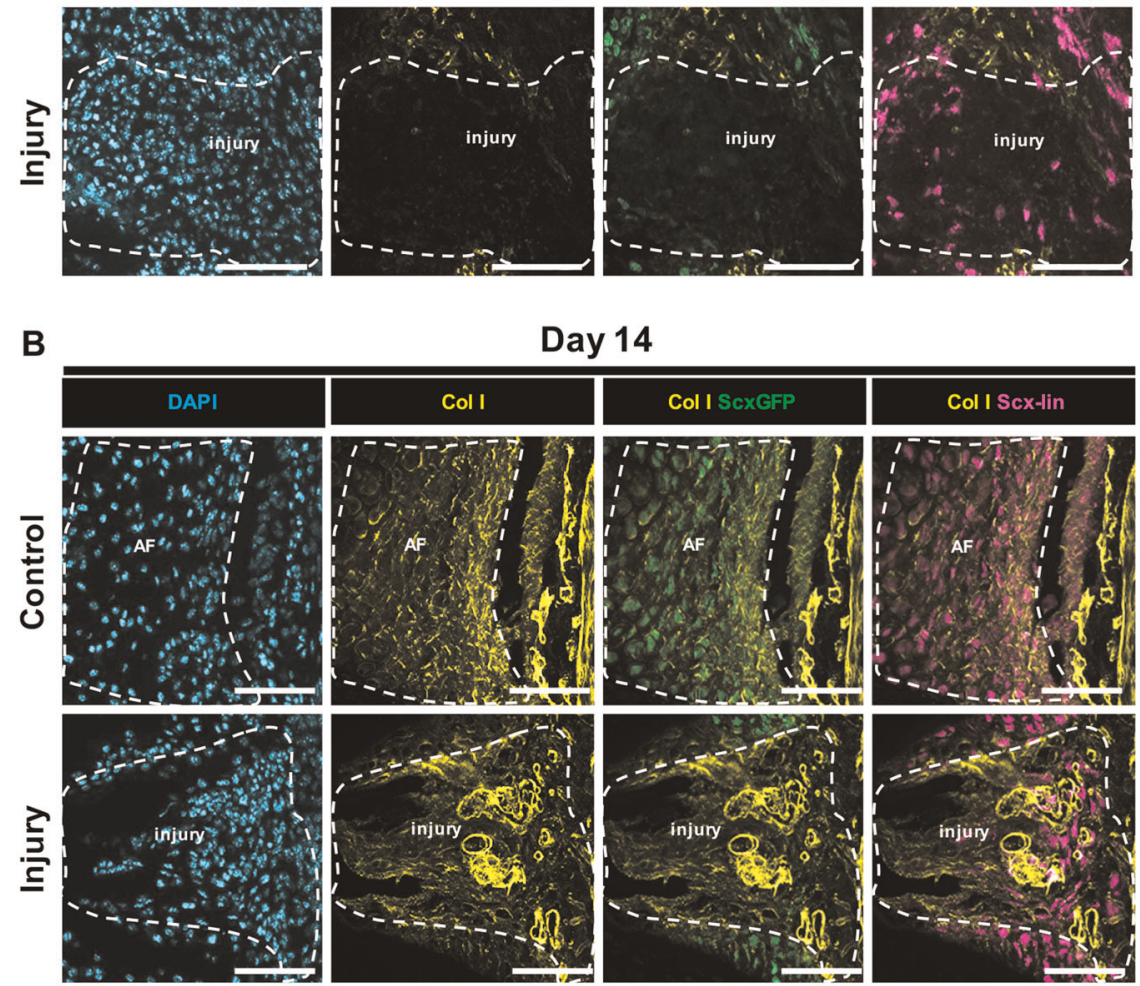

C

Day 56

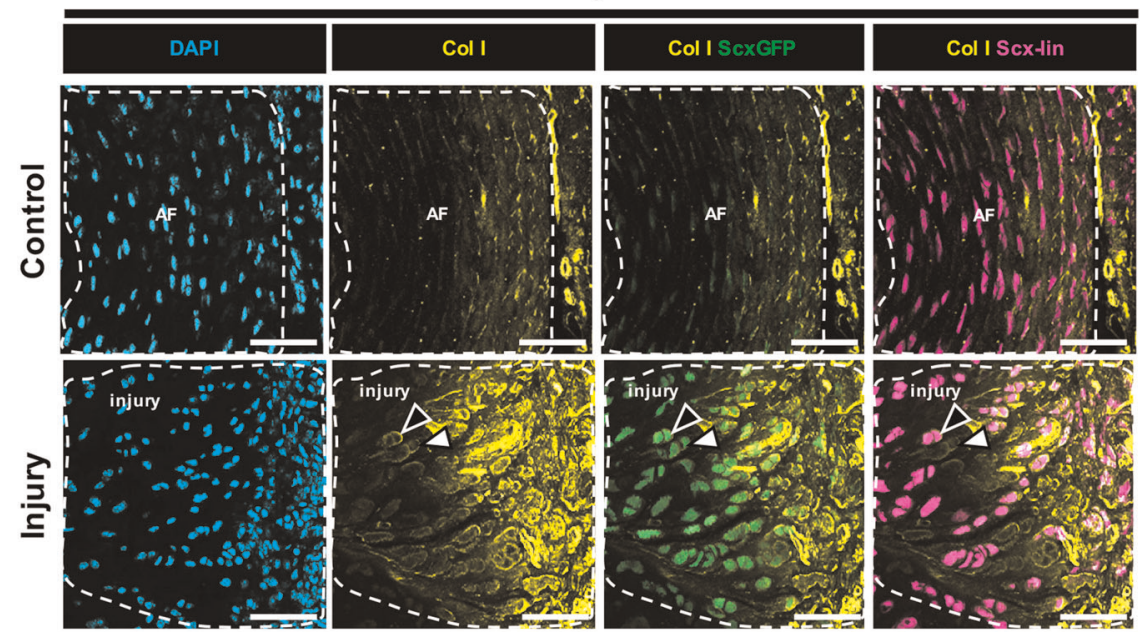

Fig. 6 Differentiated SCXGFP annulocytes produce type I collagen. Immunostaining for Col I revealed that Scx-lin cells in the injury site at d3 were ScxGFP- and did not express Col I a. At d14, Scx-lin cells colocalized with Col I b. By d56, ScxGFP expression was restored and was colocalized with Col I c. Subpopulations of Col I expressing cells were observed at d56 including cells that were Scx-lin and ScxGFP+ (black triangles) and cells that were non Scx-lin and ScxGFP+ (white triangles). ScxGFP expression in annulocytes is lost immediately following neonatal injury but annulocyte phenotype is likely restored by d56 as evidenced by colocalization of SCXGFP expression and Col I. Scale = $100 \mu \mathrm{m}$. 
A

ScxCreERT2/RosaT

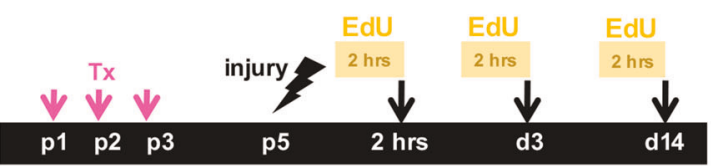

B

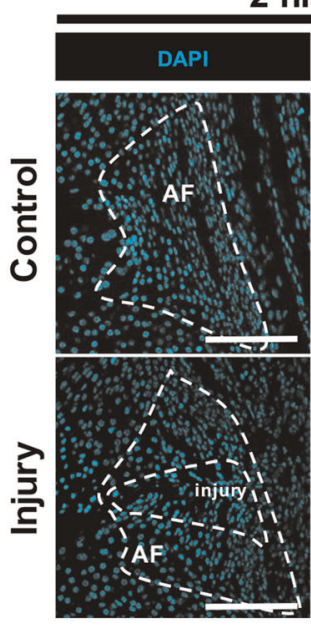

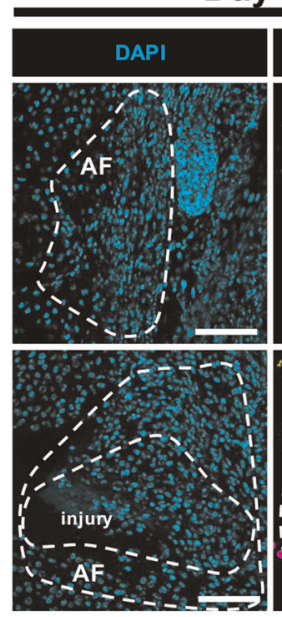

C

Day 3

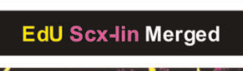

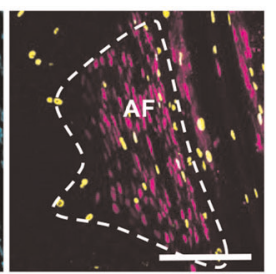
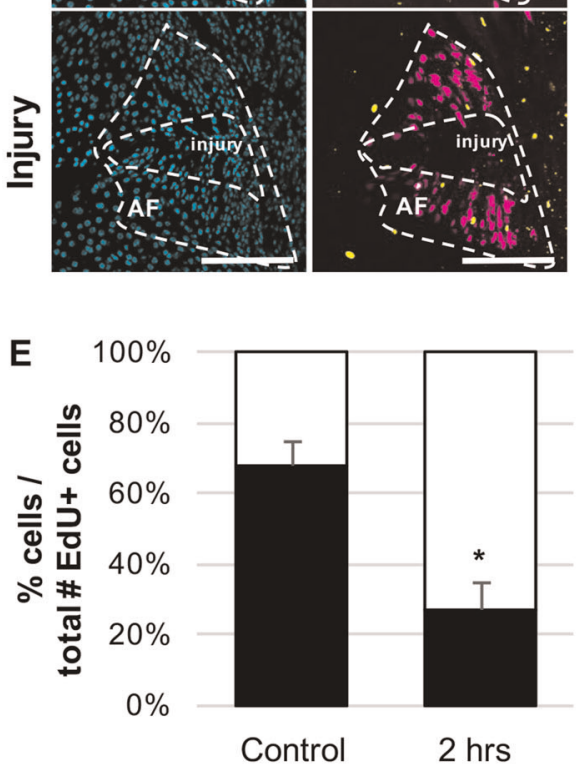

F

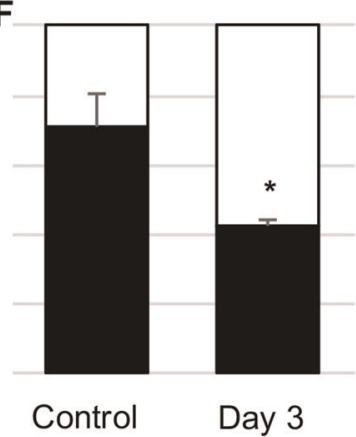

D

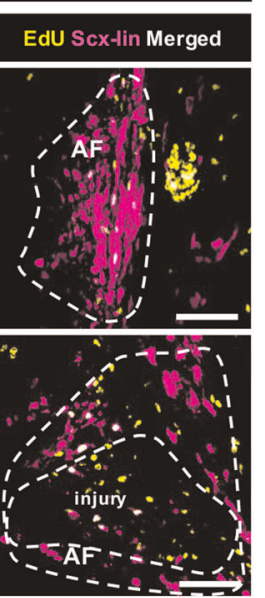

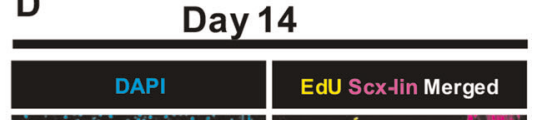
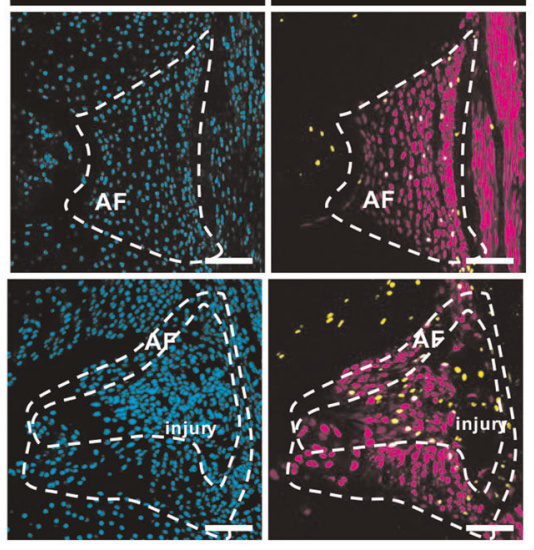

G

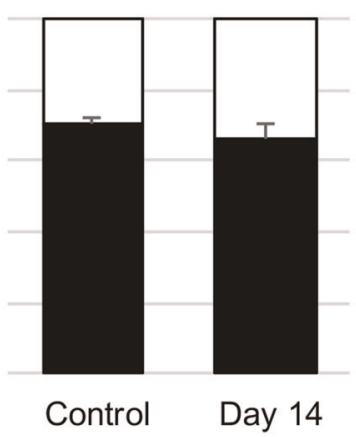

Scx-lin

Non Scx-lin

Fig. 7 Scx-lin annulocyte proliferation contributes to neonatal AF healing. EdU detection of proliferating cells in tamoxifen-labeled controls and injured ScxCreERT2/RosaT/ScxGFP mice was performed $2 \mathrm{hrs}$ prior to sac at $2 \mathrm{hrs,} \mathrm{d3}$, and d14 following herniation injury a. In uninjured controls, most proliferating cells $(\sim 60-70 \%)$ are $S c x$-lin cells (EdU $+/ S c x$-lin). With injury, the percentage of non $S c x$-lin proliferating cells is increased at $2 \mathrm{hrs}$ and $\mathrm{d} 3 \mathbf{b} \mathbf{b}, \mathbf{c}, \mathbf{e}, \mathbf{f}$, and is restored compared to control levels at d14 $\mathbf{d}, \mathbf{g}$. Error bars $=\mathrm{SD}$. Scale $=100 \mu \mathrm{m}$.

be due to the mechanically challenging environment and high tissue density, or relatively slow transport and metabolism of the relatively large and avascular IVD relative to other tissues. Nevertheless, our previous findings demonstrate that the neonatal AF regenerates following injury with restored biomechanical function. ${ }^{19}$

Diverse cellular mechanisms have been identified for regenerative tissues; these include transdifferentiation of neighboring support cells (neonatal cochlear hair cells), ${ }^{16}$ differentiation of dedicated stem cell populations (bone, muscle), ${ }^{28,29}$ blastema formation (neonatal digit tip), ${ }^{17}$ and expansion of differentiated cells retaining mitotic potential (neonatal heart, tendon). ${ }^{15,18}$ Here, we find that functional neonatal IVD regeneration is mediated by Scx-lin annulocytes with mitotic potential, as well as recruited non Scx-lin cells. Proliferation was also observed in regions adjacent to the injury site, consistent with previous studies showing cell proliferation within and adjacent to the injury site in adult salamander and neonatal mouse heart regeneration models. ${ }^{30,31}$ The presence of non Scx-lin annulocytes could be the result of incomplete tamoxifen recombination efficiency, but may also indicate a potential contribution from tissue-resident stem/ progenitor cells that are activated and differentiate following injury. Similarly, recruited Scx-lin cells may be derived from a subpopulation of stem/progenitor annulocytes. Although resident AF stem/progenitor cells have been identified in adult humans and several other species, ${ }^{32-37}$ there is still no consensus in the field on definitive markers for these cells or their location/ potential. Although nucleostemin expressing progenitors were previously identified in rabbit $A F, 38,39$ we did not observe positive staining in the neonatal mouse IVD. Similarly, CD146+ cells were also not detected. These differences may be due to differences across species or age. Interestingly, Sca-1 was strongly detected in the injured neonatal AF immediately following injury, and restored Scx-lin cells appeared to adopt a stem/progenitor phenotype as part of the regenerative healing response. In neonatal controls, Sca-1 cells were observed adjacent to the outer AF and surrounding tendons; these cells may be a source of non Scx-lin stem/progenitors observed following neonatal injury. In tendons, 
A ShhCre/RosaT

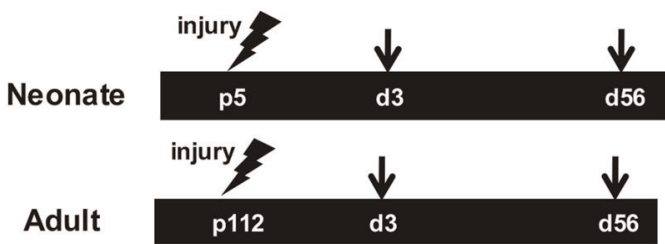

B

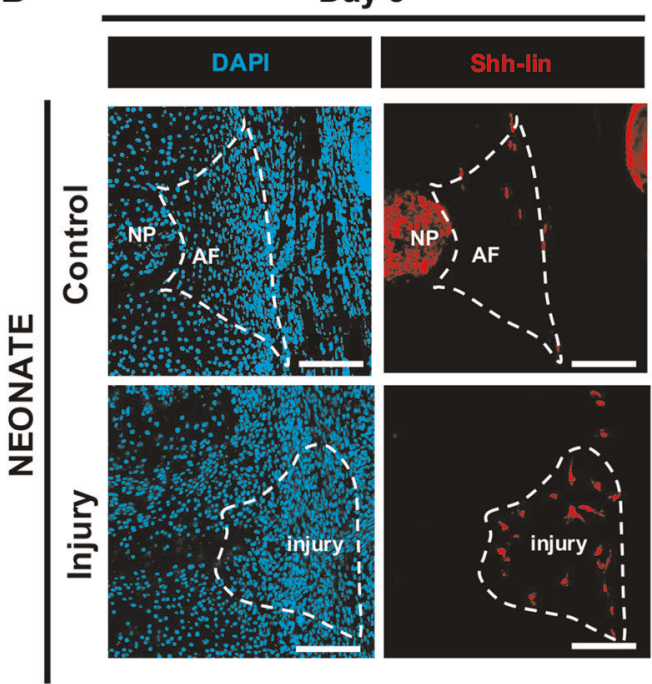

D

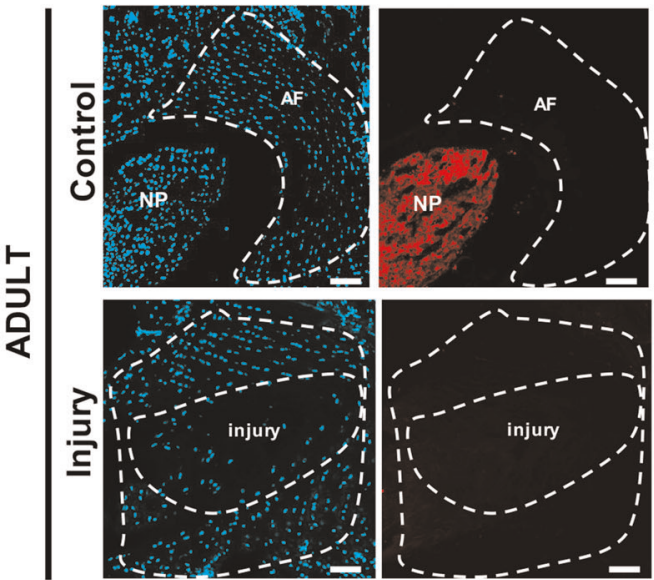

C
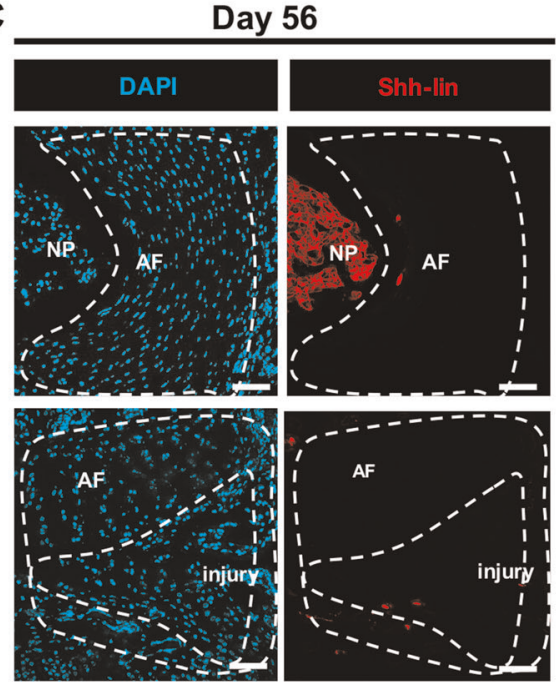

E
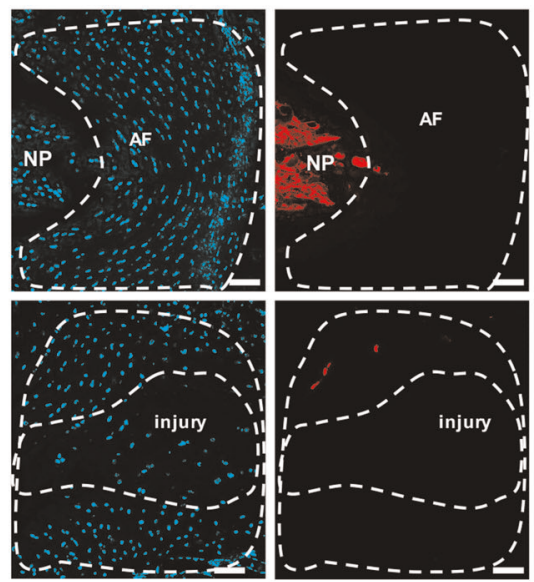

Fig. 8 Shh-lineage cells are transiently recruited to the AF injury site. Notochordal-derived, nucleus pulposus cells were traced using the constitutive ShhCre/RosaT reporter mouse. Shh-lin cells were observed in the NP region of uninjured neonatal and adult controls, and sporadically in the uninjured neonatal AF at d3 b and d56 c, e. Early recruitment of Shh-lin cells with stellate morphology was observed at the neonatal d 3 injury site b. Few Shh-lin cells were observed in the neonatal d56 injury site c. In adults, no contribution of Shh-lin cells was observed at d3 d, or d56 e. Scale $=100 \mu \mathrm{m}$.

contributions of Sca-1 cells migrating into injured tendon from the paratenon may play a role in wound healing responses. ${ }^{40}$ An abundance of Scx-lin, Sca-1 expressing cells at the d14 injury site, together with the absence of ScxGFP or Col I suggested that some $\mathrm{AF}$ cells in the injury site adopted a stem/progenitor state. Further, Sca-1 and ScxGFP expression were not mutually exclusive at d56. While this may confirm that SCXGFP is not a pure AF differentiation marker (indeed, ScxGFP labels tendon and AF progenitors at early embryonic stages ${ }^{22}$ ), the presence of Col I in the injury site suggests that cells at d56 were observed during a transition stage of differentiation towards an annulocyte phenotype. Sca-1 expression has been used to identify IVD stem/progenitor cells, ${ }^{41}$ but has also been reported in differentiated cell types. ${ }^{42}$ However, the poor regenerative capacity of the adult AF could also suggest that true stem/progenitor cells may not exist for the AF. The identification of robust AF stem/progenitor markers could address this intriguing possibility, enable mechanistic studies that test the regenerative capacity of these cells in vivo, and open novel therapeutic avenues for annulocyte repair. Of note, we employ the term "stem/progenitor" to broadly encompass both stem cells and 
progenitor cells, which are distinct cell types; stem cells are multipotent and with unlimited self-renewal capacity, and progenitor cells are unipotent and proliferative, with limited selfrenewal capacity. ${ }^{43}$ Therefore, in the absence of additional markers to distinguish the two distinct populations, Sca-1 cells observed in this study may fit either criteria.

In this study, we used ScxGFP to identify differentiated ScxGFP annulocytes and showed that SCXGFP expression declines normally with $\mathrm{AF}$ maturation and may be associated with impaired adult healing. Although $S c x$ is a robust marker for annulocytes from embryonic through postnatal stages, ${ }^{19,22,44} S c x$ null mutants do not display a significant AF phenotype, ${ }^{45}$ suggesting it is not required for AF development. Intriguingly, the SCx null phenotype is largely limited to long-range tendons throughout the body; short-range tendons, ligaments, and AF are not affected. This suggests that while $S c x$ is a consistent marker of all these fibrous cell types, $S c x$ function may be highly distinctive between tissues. Elucidating these distinct functions of $S c x$ may reveal key developmental processes that distinguish these tissues. In addition to $\operatorname{Scx}$, Sox 9 is another interesting marker for annulocytes. All annulocytes originate from the Sox9-lineage and conditional deletion of Sox9 in Scx+ cells results in aberrant AF development. ${ }^{44}$ To date, the function of Scx and Sox9 in AF regeneration and healing has not been established. For tendon, Scx function is required for matrix deposition after injury but not cell migration. ${ }^{40}$ Identifying the function of these transcription factors in AF healing will be the focus of future studies.

This neonatal AF model of functional regeneration captures key events of reparative regeneration including ScXGFP annulocyte differentiation, functional biomechanical restoration, and IVD height recovery. We demonstrated that restored ScxGFP cells in neonates colocalized with type I collagen, further supporting the conclusion that these cells may be redifferentiated annulocytes. Interestingly, we noted that Col I signal in the regenerated neonatal AF was markedly more intense than in the uninjured AF. Unmasking using hyaluronidase digestion was required to detect Col I in d56 control IVDs (Supplemental Fig. 5) and it is possible that additional unmasking or enzymatic digestion would be required to address low immunofluorescence signal that was detected in controls. It is also possible that the neonatal injury response induces increased $\mathrm{Col} \mathrm{I}$ production and remodeling. Interestingly, Col I and a-SMA cells were detected that appeared to form circular structures resembling blood vessels at the intermediate d14 timepoint. However, a marker for endothelial cells (PECAM), which is indicative of vasculature, was not detected. The presence of these structures was transient, suggesting some remodeling occurring at the injury site. However, the hierarchical and lamellar structure of the AF is not restored, suggesting a limitation to neonatal healing. Despite permanent loss of organization within the puncture site, the regions adjacent to the injury site retained its intact lamellar structure and ScxGFP expression. This suggests that puncture of neonatal IVD did not trigger the same degenerative cascade that is observed in adults. Developmentally, annulocyte differentiation and alignment precedes collagen fibrillogenesis. ${ }^{46}$ Therefore, in our neonatal model, either ScxGFP annulocytes have not realigned by the $d 56$ timepoint, or the signals that drive annulocyte alignment and organization are absent. Since healing is also influenced by the local inflammatory environment, ${ }^{30,47}$ the surrounding matrix, the mechanical microenvironment, and other factors, ${ }^{23}$ it is not surprising that AF regeneration does not fully recapitulate developmental processes.

In contrast to neonates, adult annulocytes were quiescent after injury and minimal proliferation or cell recruitment were observed overall. It is well known that the adult human IVD has limited capacity for endogenous repair and regeneration. This may be due to the loss in proliferative capacity with maturation. ${ }^{23}$ In addition to repair cells that deposit granulation tissue, immune cells such as pro-inflammatory macrophages, $\mathrm{T}$ lymphocytes, and mast cells have been implicated in poor adult AF healing. ${ }^{24,48-51}$ However, mechanistic studies testing the requirement of these cells in healing have not been carried out and the sources of fibrotic repair cells remain largely unidentified. Fibronectin is a fibrotic marker associated with IVD degeneration and has been previously identified via immunostaining in degenerated human IVD tissue. ${ }^{52}$ Although we expected increased $F n 1$ in adults after injury consistent with our observation of poor adult healing, gene expression results overall were highly variable and we found no changes in $F n 1$ expression in neonates or adults after injury. Our inability to detect differences associated with injury and regeneration/degeneration is likely due to the use of the whole IVD for this assay. The use of the whole IVD may not be sensitive enough to reflect histological findings observed at the injury site, which is a small region proportional to the entire IVD. Assessment of AF-specific differentiation and maturation genes at the injury site itself may provide more insight in to the timing of loss of AF phenotype and differentiation, but was considered outside this study's scope since such localized cell measurements (by laser capture or RNAScope) will require substantial methodological development.

Contrary to the hypothesis that herniated NP cells are retained during neonatal AF healing, Shh-lin cells do not significantly contribute to neonatal AF healing. The presence of sporadic Shhlin cells in the control AF was an unexpected finding and has not been previously reported, and is one explanation for the presence of Shh-lin cells that were observed in AF regions adjacent to the injury site of some neonatal and adult samples. Labeling of Shh-lin cells has been previously observed in the NP and in some cells of the cartilaginous endplate, but not in the AF. ${ }^{53}$ Also unexpectedly, Shh-lin cells with non-NP cell morphology were observed at d3 in the neonatal injury site. The constitutive ShhCre/RosaT mouse labels any cell expressing ShhCre with no temporal labeling control, therefore multiple interpretations of these findings are possible. The Shh-lin cells observed at the d3 injury site may be an expansion of AF-resident Shh-lin cells that were observed in controls, or Shh-lin cells recruited from an extrinsic non-NP source. Potential wound healing cells including pericytes were not detected during neonatal healing, however macrophages were observed $2 \mathrm{hrs}$ immediately after injury, indicating a rapid inflammatory response. Macrophages are likely significant mediators of early IVD healing and are required for regeneration in diverse contexts, including neonatal heart regeneration and axolotl limb regeneration. ${ }^{30,47}$ The role of macrophages and the immune response in neonatal AF regeneration warrants further investigation.

One important limitation in our model is the use of a severe herniation injury of healthy IVDs, with percutaneous laceration through several layers of skin, fascia and connective tissue, as well as complete loss of NP. This is less representative of the clinical scenario where herniation is a result of accumulated degenerative damage to the AF and typically does not involve extrusion of the entire NP. It is also possible that the needle puncture itself results in displacement of cells and tissues from surrounding structures into the injury site. However, given that most cells involved in wound healing appeared to be $S c x$-lin, we do not expect that displacement of surrounding cells and tissues resulting from the injury is a primary mechanism driving neonatal healing. Even with this severe injury, the neonatal IVD is remarkably able to restore biomechanical function and ScxGFP+ annulocytes at the injury site. A more minor injury, which is extremely technically challenging because of the small size of the neonatal mouse IVD, might enable more complete regeneration.

A significant challenge in developing cellular AF repair strategies is the lack of consensus and understanding regarding the cellular composition and cellular phenotypes of cells residing in the AF. The current study contributes to a more thorough 


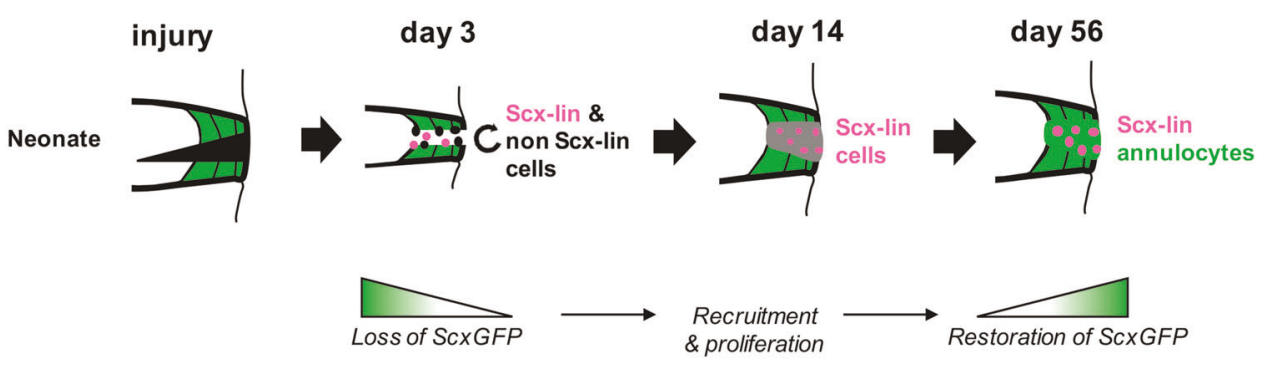

Fig. 9 Model of neonatal AF healing. Following neonatal IVD herniation injury, healing was characterized by early activation and proliferation of both Scx-lin and non Scx-lin annulocytes, some of which are Shh-lin. At d14, recruitment of Scx-lin annulocytes is observed and their proliferation continues, but cells are not ScxGFP+ at this intermediate time. By d56, there is differentiation of annulocytes that are mostly Scxlin. This process is mediated by loss of ScxGFP expression, recruitment and proliferation of Scx-lin cells, and differentiation of ScxGFP annulocytes between d14 and d56.

understanding of annulocyte subpopulations and regulators of annulocyte differentiation and organization, to help inform choices of appropriate AF cell types and strategies for repair. Here, we demonstrated that functional neonatal AF regeneration is mediated by heterogeneous cell populations that are primarily Scx-lin, and that restoration of ScxGFP + annulocytes occurs by a series of cellular events including loss of ScxGFP expression, proliferation, and redifferentiation (Fig. 9). Our neonatal model of functional AF regeneration holds promise for establishing key cellular populations and molecular signals that regulate annulocyte differentiation and regeneration. Elucidating the biology of regenerative IVD healing will enable novel therapeutic strategies for human IVD repair.

\section{METHODS}

Mice

Existing mouse lines were used in these studies including the ScXGFP reporter, ${ }^{22}$ ScxCreERT2 (generated by Dr. Ronen Schweitzer), ShhCre reporter, ${ }^{54}$ and Ai14 Rosa26-TdTomato Cre (RosaT) reporter. ${ }^{55}$ All transgenic mice are primarily of C57BL/6 background. Lineage tracing of SCxCreERT2 mice was performed by tamoxifen delivery via gavage in neonates $(1.25 \mathrm{mg} /$ pup for 3 consecutive days, followed by 2 days rest) or intraperitoneal injection in adults $(100 \mathrm{mg} / \mathrm{kg}$ wt for 5 consecutive days followed by 2 days rest) prior to injury. EdU $(0.05 \mathrm{mg})$ was delivered subcutaneously $2 \mathrm{hrs}$ prior to harvest to label proliferating cells. All animal procedures were approved by the Institutional Animal Care and Use Committee and Icahn School of Medicine at Mount Sinai and are consistent with animal care guidelines.

\section{AF injury model}

The neonatal and adult IVD injury models were previously established. ${ }^{19}$ For neonates, IVDs were localized via ScXGFP expression visualized under fluorescence directly through the skin with a stereomicroscope with fluorescence capabilities (M165FC; Leica Microsystems), followed by full AF puncture with a 31-gauge beveled syringe needle tip with tissue-marking dye (TBS). Full AF injury was performed to a depth of $50 \%$ of the dorsallateral - ventral-lateral IVD diameter to induce herniation of NP tissue (Fig. 1a). After injury, neonatal skin was not sutured because of the small size of the injury, and the animals were returned to full cage activity. For adults, IVDs were localized by palpation and exposed by blunt dissection using forceps. A 2-4 mm incision was made in the skin of the dorsolateral aspect of the tail. IVD exposure was visually confirmed under an M60 microscope (Leica Microsystems), followed by full AF puncture with a 26-gauge beveled syringe needle tip, corresponding to $80 \%$ of the IVD height (15), with tissue marking dye (TBS). After injury, skin was closed with Prolene 8-0 sutures (Ethicon), and animals returned to full cage activity. Injured and control IVDs were harvested at d0, d3, d14, d28, and d56, for histologic assessment and at $\mathrm{d} 3$ and $\mathrm{d} 56$, for gene expression studies.
RNA isolation, reverse transcription, and qRT-PCR

Total RNAs were extracted from uninjured control or injured whole IVDs after neonatal injury using Trizol/chloroform and quantified using NanoDrop 2000. Reverse transcription was carried out using SuperScript VILO Master Mix and qRT-PCR performed using SYBR Green PCR Master Mix (Thermo Fisher). Primer sequences used are listed in Supplemental Table 1. RNA samples were prepared from three injured caudal levels (c4/5, c6/7, c8/9) per animal, with 5-6 animals per group, and RNA from individual IVD levels were run as independent samples in triplicate.

Histology and immunofluorescence

For histology, neonatal (p5) and adult (p112) mice caudal level (c) 4/5 was injured and $c 5 / 6$ was used as an internal control with 3 animals per group. For fluorescence imaging and immunofluorescence of sections, tail segments were fixed in $4 \%$ paraformaldehyde and frozen in OCT medium. Alternating sagittal cryosections $(12 \mu \mathrm{m})$ were collected to capture the IVD from outer AF to the opposite outer AF. Immunostaining was carried out using antibodies against Sca-1 (R\&D Systems), type I collagen (Abcam), nucleostemin (Neuromics), F4/80 (Affymetrix), a-SMA (Sigma), PECAM (CD31) (BD Pharmingen), MCAM (CD146) (Santa Cruz), and CK19 (Abcam), with DAPI counterstaining (1:1000) to visualize cell nuclei. Primary and secondary dilutions used are listed in Supplemental Table 2. EdU and TUNEL assays were performed using the Click IT EdU and Click IT TUNEL kits (Life Technologies), according to manufacturer's instructions with appropriate positive controls (Supplemental Fig. 4). All images were acquired using Zeiss Axio Imager microscope; an Apotome was used for optical sectioning of fluorescent images.

\section{Quantification of cell populations}

Three consecutive sections, $72 \mu \mathrm{m}$ apart, were imaged from the midsagittal region, which was determined by the measurer, and the AF and injury site regions were outlined (Fig. 4b). For proliferation and TUNEL measurements at $2 \mathrm{hrs}, \mathrm{d} 3$, and d14, the entire posterior AF was quantified. For d56 measurements, only the injury site was quantified. Cell counting was performed in Image $\mathrm{J}$, and the average of three consecutive sections per disc was obtained for each cell population of interest.

\section{Statistics}

All statistical analyses were performed in GraphPad Prism with $p<$ 0.05 significance. One-tailed Student's t-test determined differences in percentage of proliferating cells and levels of cell apoptosis between control and injury in neonates, differences in the total \# of cells recruited to the injury site and percentages of different cell populations between neonates and adults, and percentages of proliferating cells between control and injury in neonates. One-tailed Student's t-test determined effect of treatment (control and injury) within each timepoint (d3 and d56) on gene expression changes.

\section{Reporting summary}

Further information on research design is available in the Nature Research Reporting Summary linked to this article. 


\section{DATA AVAILABILITY}

The data that support the findings of this study are available from the corresponding author upon reasonable request.

Received: 29 April 2019; Accepted: 25 November 2019; Published online: 20 December 2019

\section{REFERENCES}

1. Key, J. A. \& Ford, L. T. Experimental intervertebral-disc lesions. J. Bone Jt. Surg. Am. 30A, 621-630 (1948).

2. Hampton, D., Laros, G., McCarron, R. \& Franks, D. Healing potential of the anulus fibrosus. Spine 14, 398-401 (1989).

3. Smith, J. W. \& Walmsley, R. Experimental incision of the intervertebral disc. J. Bone Jt. Surg. Br. 33-B, 612-625 (1951).

4. Hagan, C. Barricaid ${ }^{(\mathrm{R})}$ Anular Closure Device (2017).

5. Carragee, E. J., Han, M. Y., Suen, P. W. \& Kim, D. Clinical outcomes after lumbar discectomy for sciatica: the effects of fragment type and anular competence. $J$. Bone Jt. Surg. Am. 85, 102-108 (2003).

6. Carragee, E. J. et al. 2009 ISSLS Prize Winner: Does discography cause accelerated progression of degeneration changes in the lumbar disc: a ten-year matched cohort study. Spine 34, 2338-2345 (2009).

7. Abdu, R. W. et al. Reoperation for recurrent intervertebral disc herniation in the spine patient outcomes research trial: analysis of rate, risk factors, and outcome. Spine 42, 1106-1114 (2017).

8. Bollman, J. L. \& Mann, F. C. Experimentally produced lesions of the liver. Ann. Intern. Med. 5, 699 (1931).

9. Michalopoulos, G. K. \& DeFrances, M. C. Liver regeneration. Science 276, 60-66 (1997).

10. Marzona, L. \& Pavolini, B. Play and players in bone fracture healing match. Clin. Cases Min. Bone Metab. 6, 159-162 (2009).

11. Knight, M. N. \& Hankenson, K. D. Mesenchymal stem cells in bone regeneration. Adv. Wound Care (N. Rochelle) 2, 306-316 (2013).

12. Mauro, A. Satellite cell of skeletal muscle fibers. J. Biophys. Biochem. Cytol. 9, 493-495 (1961).

13. Wang, Y. X. \& Rudnicki, M. A. Satellite cells, the engines of muscle repair. Nat. Rev. Mol. Cell Biol. 13, 127-133 (2011).

14. lismaa, S. E. et al. Comparative regenerative mechanisms across different mammalian tissues. npj Reg. Med. 3, 6 (2018)

15. Porrello, E. R. et al. Transient regenerative potential of the neonatal mouse heart Science 331, 1078-1080 (2011).

16. Cox, B. C. et al. Spontaneous hair cell regeneration in the neonatal mouse cochlea in vivo. Development 141, 816-829 (2014).

17. Lehoczky, J. A., Robert, B. \& Tabin, C. J. Mouse digit tip regeneration is mediated by fate-restricted progenitor cells. Proc. Natl Acad. Sci. USA 108, 20609-20614 (2011).

18. Howell, K. et al. Novel model of tendon regeneration reveals distinct cell mechanisms underlying regenerative and fibrotic tendon healing. Sci. Rep. 7, 45238 (2017).

19. Torre, O. M., Das, R., Berenblum, R. E., Huang, A. H. \& latridis, J. C. Neonatal mouse intervertebral discs heal with restored function following herniation injury. FASEB J. 32, 4753-4762 (2018)

20. latridis, J. C., Michalek, A. J., Purmessur, D. \& Korecki, C. L. Localized intervertebra disc injury leads to organ level changes in structure, cellularity, and biosynthesis. Cell Mol. Bioeng. 2, 437-447 (2009).

21. Guterl, C. C. et al. Challenges and strategies in the repair of ruptured annulus fibrosus. Eur. Cell Mater. 25, 1-21 (2013).

22. Pryce, B. A., Brent, A. E., Murchison, N. D., Tabin, C. J. \& Schweitzer, R. Generation of transgenic tendon reporters, ScxGFP and ScxAP, using regulatory elements of the scleraxis gene. Dev. Dyn. 236, 1677-1682 (2007)

23. Dahia, C. L., Mahoney, E. J., Durrani, A. A. \& Wylie, C. Postnatal growth, differentiation, and aging of the mouse intervertebral disc. Spine 34, 447-455 (2009).

24. Torre, O. M., Mroz, V., Bartelstein, M. K., Huang, A. H. \& latridis, J. C. Annulus fibrosus cell phenotypes in homeostasis and injury: implications for regenerative strategies. Ann. N. Y Acad. Sci. 1442, 61-78 (2019).

25. Kugler, M. C. et al. Sonic hedgehog signaling regulates myofibroblast function during alveolar septum formation in murine postnatal lung. Am. J. Respir. Cell Mol. Biol. 57, 280-293 (2017).

26. Horn, A. et al. Hedgehog signaling controls fibroblast activation and tissue fibrosis in systemic sclerosis. Arthritis Rheum. 64, 2724-2733 (2012).

27. Porrello, E. R. et al. Regulation of neonatal and adult mammalian heart regeneration by the miR-15 family. Proc. Natl Acad. Sci. USA 110, 187-192 (2013).

28. Sambasivan, R. \& Tajbakhsh, S. Adult skeletal muscle stem cells. Results Probl. Cell Differ. 56, 191-213 (2015).
29. Walmsley, G. G. et al. Stem cells in bone regeneration. Stem Cell Rev. 12, 524-529 (2016).

30. Godwin, J. W., Debuque, R., Salimova, E. \& Rosenthal, N. A. Heart regeneration in the salamander relies on macrophage-mediated control of fibroblast activation and the extracellular landscape. npj Reg. Med. 2, 22 (2017).

31. Notari, M. et al. The local microenvironment limits the regenerative potential of the mouse neonatal heart. Sci. Adv. 4, eaao5553 (2018).

32. Risbud, M. V. et al. Evidence for skeletal progenitor cells in the degenerate human intervertebral disc. Spine 32, 2537-2544 (2007).

33. Brown, S. et al. Cell clusters are indicative of stem cell activity in the degenerate intervertebral disc: can their properties be manipulated to improve intrinsic repair of the disc? Stem Cells Dev. 27, 147-165 (2018).

34. Henriksson, $\mathrm{H}$. et al. Identification of cell proliferation zones, progenitor cells and a potential stem cell niche in the intervertebral disc region: a study in four species. Spine 34, 2278-2287 (2009).

35. Wang, H. et al. Distinguishing characteristics of stem cells derived from different anatomical regions of human degenerated intervertebral discs. Eur. Spine J. 25 2691-2704 (2016).

36. Feng, G. et al. Multipotential differentiation of human anulus fibrosus cells: an in vitro study. J. Bone Jt. Surg. Am. 92, 675-685 (2010).

37. Yasen, $M$. et al. Changes of number of cells expressing proliferation and progenitor cell markers with age in rabbit intervertebral discs. Acta Biochim. Biophys. Sin. (Shanghai) 45, 368-376 (2013).

38. Sang, C., Cao, X., Chen, F., Yang, X. \& Zhang, Y. Differential characterization of two kinds of stem cells isolated from rabbit nucleus pulposus and annulus fibrosus. Stem Cells Int. 2016, 8283257 (2016).

39. Liu, C. et al. Identification of rabbit annulus fibrosus-derived stem cells. PLoS ONE 9, e108239 (2014).

40. Sakabe, T. et al. Transcription factor scleraxis vitally contributes to progenitor lineage direction in wound healing of adult tendon in mice. J. Biol. Chem. 293, 5766-5780 (2018).

41. Cox, M. K. et al. Erg cooperates with TGF- $\beta$ to control mesenchymal differentiation. Exp. Cell Res. 328, 410-418 (2015).

42. Holmes, C. \& Stanford, W. L. Concise review: stem cell antigen-1: expression, function, and enigma. Stem Cells 25, 1339-1347 (2007).

43. Seaberg, R. M. \& van der Kooy, D. Stem and progenitor cells: the premature desertion of rigorous definitions. Trends Neurosci. 26, 125-131 (2003).

44. Sugimoto, Y. et al. Scx+/Sox9+ progenitors contribute to the establishment of the junction between cartilage and tendon/ligament. Development 140, 2280-2288 (2013).

45. Murchison, N. D. et al. Regulation of tendon differentiation by scleraxis distinguishes force-transmitting tendons from muscle-anchoring tendons. Development 134, 2697-2708 (2007).

46. Hayes, A. J., Isaacs, M. D., Hughes, C., Caterson, B. \& Ralphs, J. R. Collagen fibrillogenesis in the development of the annulus fibrosus of the intervertebral disc. Eur. Cell Mater. 22, 226-241 (2011).

47. Aurora, A. B. et al. Macrophages are required for neonatal heart regeneration. J. Clin. Invest 124, 1382-1392 (2014).

48. Nerlich, A. G., Weiler, C., Zipperer, J., Narozny, M. \& Boos, N. Immunolocalization of phagocytic cells in normal and degenerated intervertebral discs. Spine 27, 2484-2490 (2002).

49. Nakazawa, K. R. et al. Accumulation and localization of macrophage phenotypes with human intervertebral disc degeneration. Spine J. 18, 343-356 (2018).

50. Wiet, M. G. et al. Mast Cell-Intervertebral disc cell interactions regulate inflammation, catabolism and angiogenesis in Discogenic Back Pain. Sci. Rep. 7, 12492 (2017).

51. Shamji, M. F., Guha, D., Paul, D. \& Shcharinsky, A. Systemic inflammatory and Th17 immune activation among patients treated for lumbar radiculopathy exceeds that of patients treated for persistent postoperative neuropathic pain. Neurosurgery 81, 537-544 (2017).

52. Oegema, T. R., Johnson, S. L., Aguiar, D. J. \& Ogilvie, J. W. Fibronectin and its fragments increase with degeneration in the human intervertebral disc. Spine $\mathbf{2 5}$, 2742-2747 (2000).

53. Maier, J. A. \& Harfe, B. D. Nuclei pulposi formation from the embryonic notochord occurs normally in GDF-5-deficient mice. Spine 36, E1555-61 (2011).

54. Harfe, B. D. et al. Evidence for an expansion-based temporal Shh gradient in specifying vertebrate digit identities. Cell 118, 517-528 (2004).

55. Madisen, L. et al. A robust and high-throughput Cre reporting and characterization system for the whole mouse brain. Nat. Neurosci. 13, 133-140 (2010).

\section{ACKNOWLEDGEMENTS}

We thank Dr. Ronen Schweitzer for providing the ScxGFP and ScxCreERT2 mice, Dr. Robert Krauss for providing the ShhCre mice used for these studies, and Dr. Dirk Hubmacher for helpful discussions. We also thank the qPCR CoRE at the Icahn School of Medicine at Mount Sinai for performing $q R T-P C R$ reactions. This work was 
supported by NIH NIAMS (R01 AR064157 (JCI) and R01 AR069537 (AHH). The data in this paper were used in a dissertation as partial fulfillment of the requirements for a $\mathrm{PhD}$ degree at the Graduate School of Biomedical Sciences at Mount Sinai.

\section{AUTHOR CONTRIBUTIONS}

O.M.T., J.C.I. and A.H.H. made substantial contributions to the conception of the work, design of the work, interpretation of data, and substantively revised the manuscript. O.M.T. and V.M. and A.R.M.B. contributed to data acquisition. O.M.T. contributed to analysis of the data and drafted the manuscript. All authors critically revised the work for important intellectual content and approved the submitted version.

\section{COMPETING INTERESTS}

The authors declare no competing interests.

\section{ADDITIONAL INFORMATION}

Supplementary information is available for this paper at https://doi.org/10.1038/ s41536-019-0085-4.

Correspondence and requests for materials should be addressed to A.H.H. or J.C.I.
Reprints and permission information is available at http://www.nature.com/ reprints

Publisher's note Springer Nature remains neutral with regard to jurisdictional claims in published maps and institutional affiliations.

Open Access This article is licensed under a Creative Commons Attribution 4.0 International License, which permits use, sharing, adaptation, distribution and reproduction in any medium or format, as long as you give appropriate credit to the original author(s) and the source, provide a link to the Creative Commons license, and indicate if changes were made. The images or other third party material in this article are included in the article's Creative Commons license, unless indicated otherwise in a credit line to the material. If material is not included in the article's Creative Commons license and your intended use is not permitted by statutory regulation or exceeds the permitted use, you will need to obtain permission directly from the copyright holder. To view a copy of this license, visit http://creativecommons. org/licenses/by/4.0/.

(c) The Author(s) 2019 